\title{
Recommender systems in model-driven engineering
}

\section{A systematic mapping review}

\author{
Lissette Almonte $^{1}$. Esther Guerra ${ }^{1}$. Iván Cantador ${ }^{2}$. Juan de Lara ${ }^{1}$
}

Received: 30 December 2020 / Revised: 10 June 2021 / Accepted: 16 June 2021 / Published online: 26 July 2021

(c) The Author(s) 2021

\begin{abstract}
Recommender systems are information filtering systems used in many online applications like music and video broadcasting and e-commerce platforms. They are also increasingly being applied to facilitate software engineering activities. Following this trend, we are witnessing a growing research interest on recommendation approaches that assist with modelling tasks and modelbased development processes. In this paper, we report on a systematic mapping review (based on the analysis of 66 papers) that classifies the existing research work on recommender systems for model-driven engineering (MDE). This study aims to serve as a guide for tool builders and researchers in understanding the MDE tasks that might be subject to recommendations, the applicable recommendation techniques and evaluation methods, and the open challenges and opportunities in this field of research.
\end{abstract}

Keywords Model-driven engineering $\cdot$ Recommender systems $\cdot$ Systematic mapping review

\section{Introduction}

Recommender systems (RSs) [3] are information filtering systems that aim to predict the preferences of users for a given set of items, with the purpose of offering a typically prioritised list of potentially interesting items. RSs are widely used by commercial applications such as music and video broadcasting platforms, e-commerce sites and social networks, and they are increasingly being used to help developers with software engineering activities [113]. For example, we can find

Communicated by Timothy Lethbridge.

Lissette Almonte

lissette.almonte@uam.es

Esther Guerra

esther.guerra@uam.es

Iván Cantador

ivan.cantador@uam.es

Juan de Lara

juan.delara@uam.es

1 Modelling and Software Engineering Research Group, Universidad Autónoma de Madrid, Madrid, Spain

2 Information Retrieval Group, Universidad Autónoma de Madrid, Madrid, Spain
RSs that help in choosing appropriate third-party programming libraries [95,135], recommend API method invocations [94], suggest code refactorings [30], propose features for mobile apps [58] and assist on the evaluation of change impact analysis [18], to name a few.

Modelling is fundamental in software engineering and central to some software development approaches like model-driven engineering (MDE) [19,122]. In MDE, models are the primary assets of the development process, since they are used for analysis, validation, simulation and code generation of the applications to be built, among other activities. The rationale of MDE is to improve software quality and to reduce accidental complexity and development times [62]. Following the trend in software engineering, in recent years, there have been proposals of RSs to assist in modelling tasks $[5,26,100]$ and other activities in the MDE process $[111,119]$.

In the state-of-the-art, we find surveys of RSs and their associated techniques [65,112], which review fundamental techniques for constructing RSs. We also find literature reviews on the use of RSs for software engineering [41,78], centred on RSs for code-based activities. However, perhaps because the use of RSs for MDE is an emerging research topic, to the best of our knowledge, there are no systematic studies yet analysing how RSs can be designed and employed to assist in MDE tasks. To fill this gap, this paper presents a 
systematic mapping review that covers publications ranging from 2004 to 2020 from the main digital libraries. Our study aims to answer the following research questions (RQs):

RQ1: In which ways can recommender systems assist in the different tasks within MDE processes?

RQ2: Which recommendation techniques are most commonly used to support MDE tasks, and how are recommenders for MDE evaluated?

RQ3: What are the main opportunities in recommender systems for MDE solutions?

We have selected 66 relevant papers from an initial set of 1,456 papers and classified them under four dimensions: domain, tooling, recommendation and evaluation. For RQ1, we have found that most approaches are directed to complete and repair artefacts, and work over models. Many RSs are language-independent, while the language-dependent ones frequently target UML or process modelling notations. For RQ2, we have found that most RSs are knowledge-based followed by content-based, and offline experiments are the most common evaluation approach. Finally, for RQ3, we have found that there are hardly any RSs for model transformations or code generators; and few RSs target creating, reusing or finding artefacts. Moreover, we have identified several research opportunities including the need for effective repositories of MDE artefacts that mitigate the current lack of data; techniques for adapting RSs to the user's needs; mechanisms to exploit the crowd knowledge via collaborative filtering; the user-based evaluation of RSs within MDE; and the investigation of mechanisms for the effective integration of RSs with MDE tools and low-code platforms.

Given the increasing importance that RSs are gaining in software engineering [113], we expect a similar trend in recommenders for modelling and MDE tasks, as the increase in the number of papers on this topic during the last years shows (cf. Fig. 2). Hence, our study may be useful for tool builders and researchers to understand the tasks that can be subject to recommendations, the applicable recommendation techniques and their evaluation methods, and the open challenges in this field of research.

The remainder of this paper is organised as follows. First, Sect. 2 provides background on RSs, and Section 3 overviews the main concepts and tasks within MDE. Then, Sect. 4 describes the scope and methodology of our systematic mapping review. Next, Sect. 5 reports on existing works that describe RSs to assist in modelling tasks. Section 6 discusses the results of our review, answers the research questions, analyses threats to the validity of the study and describes open challenges and interesting research directions. Finally, Sect. 7 concludes with a summary.

\section{Recommender systems}

In this section, we first provide an overview of RSs (Sect. 2.1), then we describe the main types of recommendation techniques (Sect. 2.2), and lastly, we present the most frequent methodologies and metrics for evaluating RSs (Sect. 2.3).

\subsection{Introduction to recommender systems}

RSs are software tools and associated techniques that suggest items considered relevant for a particular user [3], usually in scenarios or applications where the space of items is very large and item search and selection are difficult or even overwhelming to the user. For this purpose, RSs explicitly or implicitly gather information about the user's preferences for a set of items (e.g. movies, songs, books or products) and subsequently use the collected information to make personalised predictions on items relevant to a target user, such as which movie to watch or which book to read next. Making use of information retrieval and machine learning techniques, RSs facilitate decision-making in domains where there are many options to choose from. Instead of requiring users to specify their interests by means of a query, these systems proactively suggest items of potential relevance to the users.

In general, RSs follow a process that encompasses three main steps:

1. Collecting relevant user information;

2. Learning from the collected information to build user profiles; and

3. Applying a heuristic function or a previously built model to select and rank the items that the user is most likely to prefer.

In order to present personalised item suggestions to a user, RSs build a user profile that captures past choices and preferences of the user. This information can be either explicitly provided by the user or implicitly inferred by the system. Explicit feedback refers to preference statements made by the users about items they know, and which are typically stored as numeric ratings or unary/binary values (e.g. likes and thumbs up/down). In contrast, implicit feedback is inferred by observing and mining user interactions within the system, such as previous search queries, product purchases and mouse clicks, among others. Other features that can be used to model the preferences of users include demographic data, personality traits, emotional states and trust relationships [112].

In a similar way, RSs characterise the items that may be recommended by means of item profiles. These profiles take different item attributes into account, such as metadata and text features extracted from item descriptions or textual contents [77]. 


\subsection{Types of recommender systems}

RSs are commonly classified into the following major categories, depending on how they generate personalised recommendations:

- Content-based systems, which recommend items that are similar to other items the target user liked in the past [77];

- Collaborative filtering systems, which base their suggestions on the items liked by "similar" people to the target user [96,120];

- Knowledge-based systems, which exploit domain knowledge to describe and relate users and items for providing personalised recommendations [22];

- Context-aware systems, which consider the current user's context (e.g. location, time, weather) to enrich personalised recommendations [4];

- Social-based systems, which analyse and exploit the social network connections of the target user to generate recommendations [46];

- Demographic-based systems, which use demographic data to represent user and item profiles considered in the recommendation generation process [102]; and

- Hybrid systems, which combine two or more of the previous types of RSs [23].

Additionally, RSs can be categorised according to the algorithmic approach they use to compute the relevance of items [3]. In this regard, we can distinguish between two types of systems:

- Memory-based systems, where the relevance of items is estimated through heuristic formulae [120]; and

- Model-based systems, which predict item relevance by using a data-based model built via machine learning techniques, e.g. matrix factorisation [70] or neural networks [48].

The following subsections explain in more detail the above categories and types of RSs, which will be considered in our review.

\subsubsection{Content-based recommenders}

Content-based (CB) systems recommend similar items to those items the target user liked in the past [3]. They use item attributes or features to represent both user and item profiles and establish the corresponding user/item similarities. In general, they consider textual information (e.g. keywords, metadata and social tags) to build the user and item profiles [77].

A CB recommender is able to provide accurate personalised suggestions when it has enough information about the target user's preferences, since content similarities can be easily established. Moreover, it is capable of suggesting items for which no preferences have been expressed yet (i.e. cold items), since recommendations are generated via contentbased item similarities.

However, this type of RSs has certain disadvantages. One of them is the overspecialisation problem, in which the user is exposed to items that are very similar to the ones the user already knows, limiting the discovery of diverse, relevant items. In this sense, CB recommenders are not suitable for domains and applications where, at a certain point, the user has to be suggested novel, fresh or even unexpected (serendipitous) recommendations, e.g. in the news articles domain. Another drawback of these systems is the new user cold-start problem, as a RS needs a considerable amount of users' preferences before it can provide well-suited recommendations.

\subsubsection{Collaborative filtering recommenders}

Collaborative filtering (CF) systems make suggestions to the target user based on items preferred by like-minded people [3]. They rely on the feedback (commonly ratings) that users give about the items. Hence, user and item similarities are established via explicit or implicit rating-based similarities and patterns $[48,120]$.

Differently to CB approaches, a CF system is able to provide novel and diverse recommendations for the target user. Even in situations of rating sparsity, CF has shown better performance than $\mathrm{CB}$ in many real-world applications and represents the most widely used approach for providing personalised recommendations [70].

Nonetheless, similarly to $\mathrm{CB}$ approaches, CF recommenders suffer from the new user cold-start problem, i.e. they need to have enough ratings to provide accurate recommendations. CF also manifests the so-called item cold-start problem, since an item can only be recommended after being rated. Moreover, CF is affected by situations of high sparsity, where the number of collected ratings is very small with respect to the total number of possible ratings given by users to items.

\subsubsection{Knowledge-based recommenders}

Knowledge-based (KB) systems recommend items using domain-specific knowledge about how item attributes and features could meet the user's needs and interests [22]. Many recommendation approaches can be categorised as KB. Among them, two types of approaches have gained great interest in the literature: case-based and constraint-based [112]. Case-based systems address the recommendation task via case-based reasoning methods, which aim to solve a new problem (i.e. a new case) by remembering previous similar 
cases and reusing knowledge about them. Constraint-based systems, on the other hand, predominantly exploit knowledge, commonly expressed by means of explicit rules, on how user requirements are related to the item attributes and features.

The main advantage of $\mathrm{KB}$ systems is their capability of providing and explaining accurate recommendations that entail a deep understanding of the user's preferences, which cannot be achieved by CB and CF approaches. Although KB systems usually do not suffer from cold-start problems, they are affected by the so-called knowledge acquisition bottleneck. This consists on the need of learning models or domain experts to model and build the used knowledge bases. Additionally, KB recommenders usually are ad-hoc solutions to particular problems, and thus their generalisation to other problems or domains is difficult or not possible.

\subsubsection{Hybrid recommenders}

Hybrid systems make use of two or more recommendation methods, such as $\mathrm{CB}$ and $\mathrm{CF}$, to take advantage of their benefits and avoid some of their limitations [23]. Because of this, many real-world RSs are hybrid. Without entering into details, we can identify three main ways to implement a hybrid system:

- Incorporating some feature of one recommendation method into another one, e.g. a CF strategy that uses CB similarities;

- Combining the recommendations generated separately by two methods, e.g. via ranking aggregation and diversification techniques; and

- Building a unifying recommendation model that incorporates characteristics of distinct methods, e.g. a matrix factorisation model with both collaborative and contentbased features.

\subsubsection{Other recommenders}

There are other types of RSs that can be considered orthogonal to $\mathrm{CB}, \mathrm{CF}$ and $\mathrm{KB}$ systems, since they exploit particular data following $\mathrm{CB}$ and $\mathrm{CF}$ strategies. Special attention can be drawn to the next recommenders:

- Context-aware recommenders, commonly abbreviated as CARS, take into consideration contextual information associated or influencing to user preferences when generating personalised recommendations [3]. Quoting Dey [32], "context is any information that can be used to characterise the situation of an entity." In CARS, context commonly refers to circumstances in which recommendations are produced, such as the time, the weather and the user's current location.
CARS are appropriate for applications where contextual variables determine or have a high impact on the relevance of the suggested items. For instance, in a RS for travelling, the vacation recommendations for the winter season can be very different from those generated for the summer [4]. Naturally, not all contextual information available might be relevant, and the fact that contextual factors and data sources differ from application to application makes CARS difficult to implement and evaluate. Moreover, CARS may require extra effort from the users, who may need to provide information about certain contextual conditions, such as their current mood and companion.

- Social-based recommenders generate personalised recommendations in social media [46]. A widely explored approach in these systems is the exploitation of explicit relationships between users in social networks. In this sense, many solutions are based on the so-called trustbased model, where the social influence and trust of users are established and propagated through the social network. In fact, research supports the theory that social trust can be used as a positive way to generate explanations of provided recommendations [133]. Social-based methods perform well when used together with other recommendation approaches, like $\mathrm{CB}$ or $\mathrm{CF}$, since social network information can help in dealing with the user and item cold-start problems [112].

- Demographic-based recommenders make use of demographic data about the users, e.g. age, gender and address [102]. Taking this information into account, the recommendation algorithms identify users or items that are demographically compatible with the target user. These systems assume that users with similar demographic attributes may rate similarly and have been applied to alleviate cold-start problems of traditional recommendation approaches.

\subsection{Evaluation of recommender systems}

RSs need to be evaluated at different phases of their lifecycle. At design time, it is necessary to assess the adequacy of the selected recommendation approach for the application at hand. This evaluation is done via offline experiments by running potential recommendation algorithms on the same dataset of user-item interactions (i.e. the rating matrix) and comparing their performance by means of several metrics [45]. This type of evaluation permits measuring the quality of the algorithms in accomplishing a recommendation task, but neglects user-centred aspects related to usage satisfaction, acceptance and experience with the system.

In offline experiments, as commonly done for evaluating machine learning methods, the dataset is usually split into two subsets: the training set, used to build the recommendation 
model, and the test set, used to evaluate the built model. Sometimes, a third subset, the validation set, is also used for parameter tuning of the trained model, before testing.

There are two main types of recommendation tasks that can be evaluated: the item rating prediction (which is in disuse in the RSs community) and the item ranking generation (or top-N recommendation). Each of them has specific metrics, sometimes adopted and adapted from the machine learning and information retrieval areas. In the rating prediction task, the objective of a RS is to accurately predict the numeric value of the rating a user would give to an item, and thus metrics such as the mean absolute error (MAE) and the root mean square error (RMSE) are considered. In the ranking generation task, the goal of a RS is to provide the user with a personalised ranked list of relevant items, with special interest in the items at the first (top) positions of the list. In this case, metrics oriented to measure the accuracy of the ranking are used, e.g. precision, recall, mean reciprocal rank (MRR) and normalised discounted cumulative gain (nDCG) [45]. These accuracy metrics can be complemented with metrics for other ranking characteristics, such as diversity, novelty and coverage [14].

RSs should also be evaluated after deployment. This can be done via online experiments, which usually are usercentric [67]. The built system is deployed in a real environment and tested by end-users in real-time, commonly online. In these experiments, a widely used evaluation methodology is $A / B$ testing, where two versions $\mathrm{A}$ and $\mathrm{B}$ of the system are deployed, and one of them implements the recommendation algorithm or functionality that is being evaluated. After a period of time, the user feedback and behaviour recorded in both systems are analysed and compared according to certain metrics. There is also the possibility of performing a user study where a prototype of the system is deployed in a controlled setting and evaluated with a reduced set of users, maybe recruited by crowd-sourcing. User studies can also follow the $\mathrm{A} / \mathrm{B}$ testing methodology and incorporate online questionnaires to gather the usage satisfaction and opinions about the system and its functionalities.

\section{Model-driven engineering}

In this section, we provide a brief overview of the main concepts, artefacts and tasks within MDE solutions. We do not aim to be exhaustive, but to provide the necessary context to understand the kind of support needed from RSs in MDE. The interested reader can see [19] for a more detailed account of MDE.

\subsection{MDE artefacts}

Figure 1 shows a schema with the main elements of MDE solutions. In MDE, models are the main assets, from which

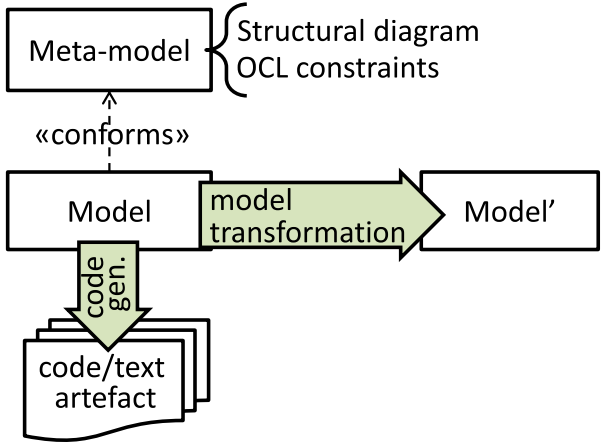

Fig. 1 Main elements of MDE solutions

other artefacts—like code or other models - may be derived in an automated way. Models conform to a meta-model, which defines the modelling language syntax and determines the set of models that are valid. Meta-models comprise a structural diagram plus additional constraints formulating restrictions that cannot be expressed diagrammatically. The structural diagram is defined as a class diagram, frequently using standards like the Meta-Object Facility (MOF) [85] and implementations like the Eclipse Modeling Framework (EMF) and Ecore [131]. Constrains are described using constraint languages like the Object Constraint Language (OCL) [97].

In addition to models, MDE solutions may include model transformations to modify existing models (e.g. performing refactorings or optimisations) or to create new models out of existing ones (e.g. creating a design model from a requirements model). Model transformations are defined using either specialised transformation languages like ATL [59] or QVT [107], general-purpose languages like Java, or technologies like XSLT [79].

Finally, textual artefacts-like code, configuration files or documentation - can be produced from models using code generators. These are typically written in specialised template languages, such as Acceleo [2] or EGL [115].

\subsection{MDE tasks}

Model-based software solutions involve the creation of models using a modelling language. Modelling languages can be either general-purpose like the UML, or domain-specific languages created for a particular domain. Therefore, in MDE, engineers may need to create the following kinds of artefacts: models, meta-models (i.e. modelling languages), model transformations, and code generators.

As in any software engineering process, in MDE, it is desirable to be able to reuse existing artefacts to avoid their creation from scratch. This requires the ability to find similar artefacts, or fragments of them, in existing repositories. 
The syntactic correctness of models is crucial to enable sound solutions and be able to apply model transformations and code generators on them. Therefore, it is important to complete partial models to become conformant to their metamodel. This completion process applies not only to models, but also to model transformations, code generators and metamodels, as these can be seen as models that have a specific semantics and conform to their own meta-models.

MDE artefacts may have errors, and so, they may need to be repaired either syntactically to conform to their meta-models, or semantically to conform to some specified requirements. Meta-model/model co-evolution [38] is a particular case of the latter, whereby a meta-model evolves to accommodate changing or new requirements, and the broken models need to be repaired to make them conform to the new meta-model version. Other tasks related to repairing artefacts include the creation of input test data (e.g. input models for testing model transformations) [13], oracles (e.g. transformation contracts) [44], and fixes [119].

\section{Survey methodology and scope}

Following accepted guidelines for systematic mappings [104, $105,139]$, we have performed a systematic mapping review to analyse how pervasive is the use of RSs to support modelling and MDE, identifying the tasks that have been subject to recommendations and the recommendation techniques most frequently applied. The surveyed articles typically introduce RSs that facilitate some modelling or MDE task.

To collect articles on this topic, we sought into Scopus, the ACM digital library and the Web of Science using a formal query comprising 23 terms. The query retrieves articles whose title, abstract or keywords contain at least one term related to RSs and at least one term related to modelling or MDE.

Table 1 shows the considered terms, so that the retrieved articles should contain in their title, abstract or keywords some term from each column of the table. We included terms like model completion, model reuse and model repair as related to RSs, because we detected that some approaches did not use standard terminology and vocabulary of the RSs area (cf. Sect. 2). However, they pursue the same goal of recommending a reduced set of modelling items or actions among a large set of possible ones. We executed the query in September 2020, and only considered peer-reviewed papers written in English and published in journals, conferences, workshops and book chapters.

Table 2 shows a summary of the search results. The query initially retrieved 1456 documents: 979 from Scopus, 316 from the ACM digital library and 161 from the Web of Science. After removing duplicates, 1175 unique documents remained.
Table 1 Terms used in the formal search query

\begin{tabular}{ll}
\hline Recommender systems/purpose & Modelling/MDE \\
\hline Recommender & Model-driven \\
Recommendation & Domain-specific language \\
Model completion & State machine \\
Model reuse & Model transformation \\
Model repair & Code generation \\
Transformation completion & Code generator \\
Transformation reuse & Unified modelling language \\
Transformation repair & UML \\
Generator completion & \\
Generator reuse & \\
Generator repair & \\
Quick fix & \\
Quick fixes & \\
Assistant & \\
Assistance &
\end{tabular}

Articles must contain in their title, abstract or keywords at least one term from each column

Table 2 Research papers retrieved per database

\begin{tabular}{ll}
\hline Detail & Num. papers \\
\hline Databases queried & 979 \\
Scopus & 316 \\
ACM & 161 \\
Web of science & \\
First revision phase & 1456 \\
Total retrieved & 1175 \\
Unique & 1024 \\
Discarded & 151 \\
First selection & \\
Second revision phase & 53 \\
Relevant & 9 \\
Not available & 89 \\
Not relevant & \\
Snowballing & 13 \\
Snowballing papers & 66 \\
Total relevant &
\end{tabular}

Next, the unique documents were filtered in two subsequent phases. In the first phase, four reviewers examined the abstracts of all documents to identify which ones proposed some kind of recommendation for modelling tasks. The reviewers were two professors specialised in MDE, one professor with expertise in RSs and information retrieval, and one doctoral student in both research areas. Overall, 151 documents were selected by at least one of the reviewers and were moved to the next phase, and 1024 were discarded for being unrelated to our study. In both phases, we used inclusion 


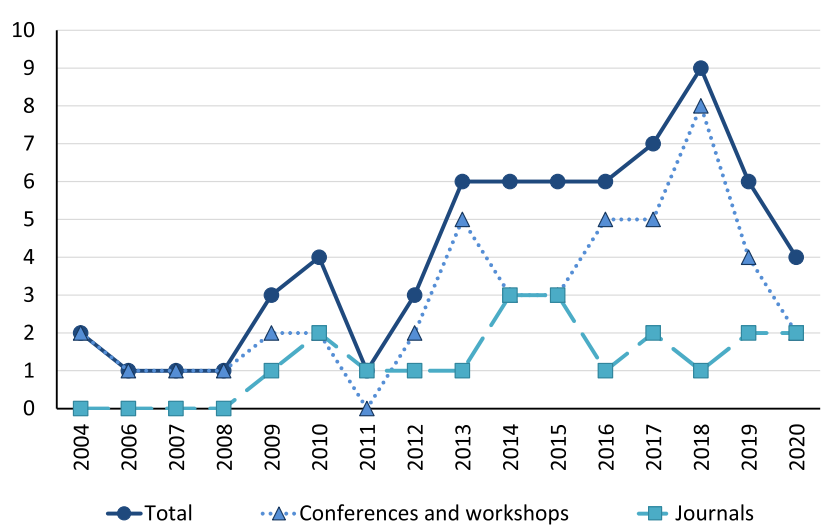

Fig. 2 Relevant papers per year

criteria based on quality (peer-reviewed papers), language (papers written in English), and focus (we discarded papers unrelated to modelling, recommenders or modelling assistants, as well as papers focussing on using MDE techniques for creating RSs).

In the second phase, the 151 selected papers were carefully read. From these papers, 53 were considered relevant for the study, 9 were unavailable, and 89 were not relevant since they either proposed RSs for activities not related to models or modelling, or did not describe recommenders or modelling assistants.

Finally, we conducted a snowballing process [105], analysing related works in the bibliography of the selected papers. This resulted in the selection of 13 additional relevant papers. Overall, a final set of 66 relevant papers was considered, ranging from 2004 to September 2020, covering almost 16 years of research. These 66 documents account for 51 different approaches, as in some cases, there are several documents covering the same approach. Figure 2 shows the distribution of papers over the studied period of time. We observe an increasing trend that likely suggests a growing interest in the field. Please note that the query may not fully cover the year 2020 as it was executed on September 2020.

Figure 3 categorises the relevant papers according to the publication type. Most papers are from conferences and workshops, which denotes that the research area is still young. The most frequent conferences and journals of publication are the International Conference on ModelDriven Engineering Languages and Systems (MoDELS) (9 papers), the International Conference on Model-Driven Engineering and Software Development (MODELSWARD) (6 papers), the International Conference on Software Engineering (ICSE) (4 papers), and the Journal on Software and Systems Modeling (SoSyM) (3 papers). Hence, the primary publication venues are devoted to software engineering and modelling.

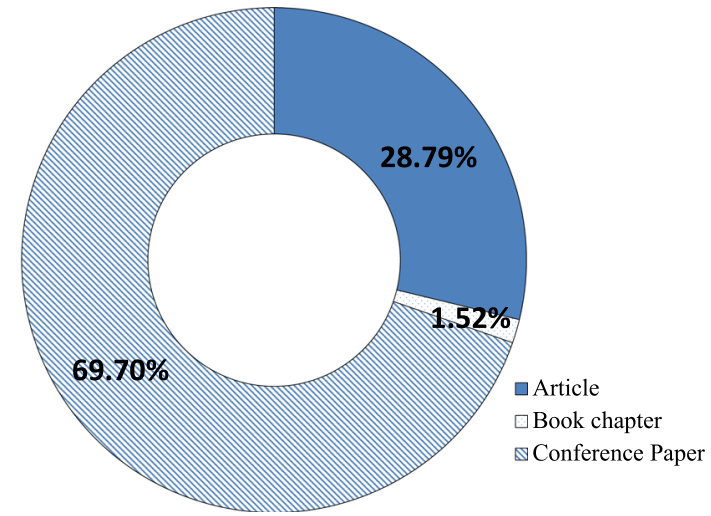

Fig. 3 Distribution of works depending on publication type

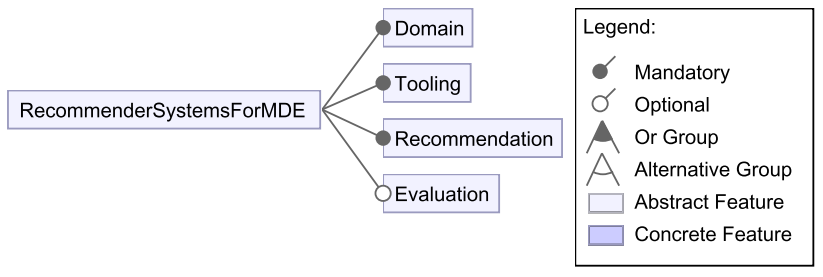

Fig. 4 Dimensions for analysing the use of RSs in MDE

In the next section, we propose a classification of the works along four dimensions and analyse the papers with respect to these categories.

\section{Recommender systems in MDE}

We organise our review according to the four dimensions of the feature model [61] depicted in Fig. 4: domain, tooling, recommendation and evaluation.

The domain dimension encompasses analysis variables in the context of MDE applications, such as the type of artefact that is subject of the recommendation and the purpose of the recommendation. The tooling dimension includes aspects related to the recommendation tool, such as its maturity, its support for the integration with other MDE tools, and its proactiveness to request or apply recommendations. The recommendation dimension entails variables used in the RSs area to characterise how recommendations are generated [106], such as the recommendation method, the user preferences used to calculate the recommendation, the recommended items and the recommendation tasks. Lastly, the evaluation dimension refers to the methodologies and metrics used to evaluate the recommenders.

These dimensions represent assessment criteria from both the MDE and the RSs perspectives. The dimensions and variables are orthogonal, although some of them may have cross-dependencies in some of the surveyed cases. For instance, the recommendation purpose may influence the 


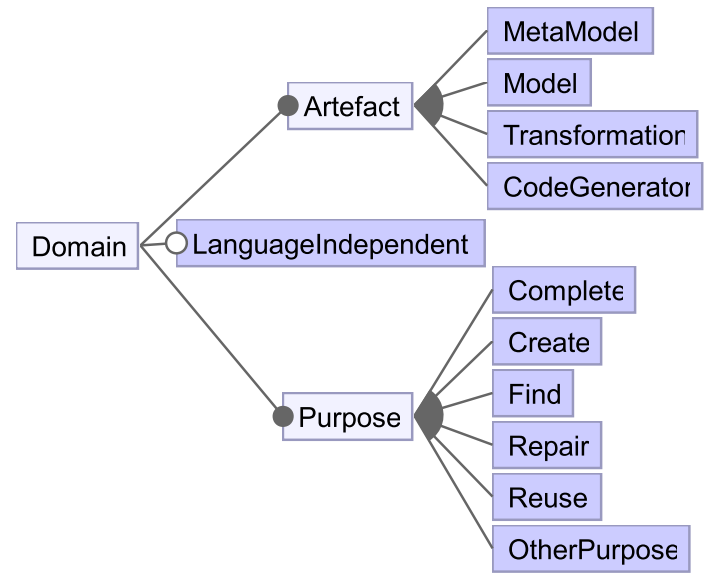

Fig. 5 Domain dimensions for RSs in MDE

metrics used to evaluate a given recommender. However, these dependencies have less impact than those between the variables belonging to a given dimension, which are addressed in the analysis presented herein.

The next four subsections analyse and classify the selected papers along the dimensions and inner variables.

\subsection{Domain}

A fundamental aspect of any RS is its application domain. This comprises the three orthogonal features that we present in the feature model of Fig. 5.

First, we consider the type of artefact that is the subject of recommendation: model, meta-model, model transformation or code generator. These are the four main elements of most MDE solutions [19].

Second, we distinguish whether the RS is languageindependent, or on the contrary, it is tied to a particular language for modelling (e.g. UML [136], Simulink [128]), meta-modelling (e.g. MOF [85], Ecore [131]), model transformation (e.g. ATL [59], QVT [107]), or code generation (e.g. Acceleo [2], EGL [115]).

Finally, we look at the purpose of the RS, that is, the kind of task that the recommender facilitates. ${ }^{1}$ As we will see later, the reviewed papers target one or more of the following six types of tasks, introduced in Sect. 3: complete, create, find, repair, reuse and other purpose. When the purpose is complete, the artefact already exists and the RS provides suggestions on how to extend it. When the purpose is create, the recommender helps in constructing the initial version of a new artefact from scratch. If the purpose is find, the RS facilitates the discovery of relevant elements or artefacts within

\footnotetext{
1 We use purpose and task interchangeably, though the latter can be more detailed. For example, for repairing (a purpose) we can find finegranular tasks, like creating input test data, oracles and selecting fixes (cf. Sect. 3).
}

a repository. Recommenders targeting repair tasks suggest solutions to fix errors in an existing artefact. These solutions may imply the creation, deletion or modification of different elements inside the artefact. When the purpose is reuse, the system helps in reusing an existing artefact (or part of it) within another artefact. This task goes beyond find as the recommender provides assistance in integrating the reused artefact in the new context. Finally, in other purpose we collect the tasks with a purpose different from the mentioned before.

Table 3 classifies the surveyed papers by purpose and artefact type, and marks the language-independent approaches with an asterisk (*). Since some systems can be used with various purposes, they can appear in several cells of the table, sometimes with different language-independent marks.

Overall, we can see that there are virtually no recommenders for code generators, and recommenders that help in creating new artefacts from scratch are also scarce. Most RSs are for models, especially for model completion and model repair, and the context in the latter case is sometimes model/meta-model co-evolution. In the following, we analyse the application domain of the approaches grouped by their purpose.

Complete. Most approaches whose purpose is completing an artefact target model completion, and among them, four are also applicable to meta-models. In addition, two approaches deal with completing model transformations. Approaches to model completion can be classified into two categories. The first one comprises techniques to recommend how to extend a partially specified model to make it correct (i.e. the recommended complete model satisfies every specified domain and meta-model well-formedness constraint). The proposed model completions are typically computed using search-based techniques, for example with solvers based on Alloy [54] (a constraint solver over models), Prolog (a logic-based programming language) or via rules. DIAGEN [82], DIG MDE [92], IPSE [40], Kermeta [86], Refacola [130] and the work by Sen et al. $[126,127]$ belong to this category. DIAGEN generates possible completions based on hypergraph grammar rules, and the others use Prolog or Alloy for this task. This search may have a high computational cost. For this reason, when a partial model cannot be completed automatically because of its complexity, DIG MDE identifies the failing constraints and suggests how to manually change the model to enable its completion. The second model completion category comprises works providing step-wise recommendations on how to evolve a given model. This model does not need to be partial, as in the first category. Suggestions usually come from repositories of existing models, fragments or pat- 
Table 3 Purpose of recommendation vs. recommended artefacts (approaches marked with * are language-independent)

\begin{tabular}{|c|c|c|c|c|}
\hline Purpose Artefact & Meta-Model & Model & Transformation & Code Generator \\
\hline Complete & $\begin{array}{c}\text { DoMoRe }[5,6] \\
\text { Hermes }[34-36] \\
\text { Kögel et al. }[68,69] \\
\text { Refacola }[130]\end{array}$ & $\begin{array}{c}\text { Baya [27] } \\
\text { Deng et al. [31] } \\
\text { DiAGEN* [82] } \\
\text { DIG MDE [92] } \\
\text { DoMoRe* [5, 6] } \\
\text { Elkamel et al. [37] } \\
\text { Heinemann [49] } \\
\text { Hermes* [34-36] } \\
\text { IPSE [40] } \\
\text { Kermeta* [86] } \\
\text { Kögel et al.* [68,69] } \\
\text { Koschmider et al. [50,51,71] } \\
\text { Li et al. [75] } \\
\text { PME* [99] } \\
\text { Rangiha et al. [110] } \\
\text { RapMOD [72,73] } \\
\text { Refacola* }[130] \\
\text { Savary-Leblanc [121] } \\
\text { Sen et al.* [126, 127] } \\
\text { SimVMA [132] } \\
\text { SMART [47] }\end{array}$ & $\begin{array}{c}\text { AXSM [52] } \\
\text { CONVErT [10] }\end{array}$ & \\
\hline Create & DSL-maps [103] & UCcheck [9] & & \\
\hline Find & Extremo [123-125] & 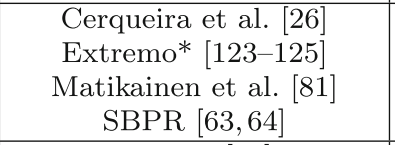 & & \\
\hline Repair & $\begin{array}{c}\text { Batot et al. }[12] \\
\text { Clarisó et al. }[28] \\
\text { PARMOREL }[11,53] \\
\text { Refacola }[130]\end{array}$ & $\begin{array}{c}\text { AMOR [21] } \\
\text { Anguel et al.* [8] } \\
\text { ASIMOV* [38] } \\
\text { BAM [137] } \\
\text { BPMoQualAssess [60] } \\
\text { B-repair [24] } \\
\text { DiAGEN* [82] } \\
\text { DPF* [108] } \\
\text { IntellEdit* [93] } \\
\text { Mani et al.* }{ }^{*}[79] \\
\text { MDSafeCer [87] } \\
\text { Nassar et al.* [91] } \\
\text { PARMOREL* [11,53] } \\
\text { Refacola* [130] } \\
\text { ReVision* [98] } \\
\text { SMART [47] }\end{array}$ & anATLyzer [117-119] & Mani et al. [79] \\
\hline Reuse & Hermes [34-36] & \begin{tabular}{|c|} 
Hermes* $^{*}[34-36]$ \\
Koschmider et al. $[50,51,71]$ \\
Paydar et al. $[100,101]$ \\
REBUILDER $[42]$ \\
SimVMA $[132]$ \\
\end{tabular} & Refactory [111] & \\
\hline Other Purpose & & $\begin{array}{l}\text { Bobek et al. [17] } \\
\text { MAGNET [16] } \\
\text { ModBud [116] }\end{array}$ & & \\
\hline
\end{tabular}

terns. For example, SimVMA [132] provides step-wise suggestions to evolve Simulink models based on model clone analysis; Heinemann [49] recommends elements defined in model libraries (e.g. blocks from Simulink libraries) based on data mining existing models; the approach by Kögel et al. [68,69] recommends model changes applicable to the same context of the last model change; DoMoRe [5,6] suggests domain concepts and names for new model elements; RapMOD [72,73] offers auto-completion actions for (UML) graphical models, similarly to the vision paper [121]; Elkamel et al. [37] recommend UML classes that are similar to the ones in the UML class diagram being developed; Li et al. [75] and Deng et al. [31] recommend activity nodes for process 
models; Rangiha et al. [110] recommend tasks and actor roles in a social process-modelling tool; Koschmider et al. $[50,51,71]$ recommend process fragments to complete a process model; Baya [27] recommends mashup model patterns based on the context, the user and different expert recommendations, and helps in weaving the selected pattern into the partial model under development; and Hermes [34-36] permits building Eclipse-based RSs that help in completing models with recommended elements from other models in a repository.

Instead of profiting from repositories of models, PME's recommendations are based on an analysis of the language meta-model [99]. PME enables proactive (graphical) modelling, meaning that plausible modifications according to the models' meta-model are automatically applied, and the user is prompted only when several optional modifications exist. SMART [47] supports the use of test-driven development to create UML diagrams (class, use cases, state machines and sequence diagrams). It uses an action language to specify behavioural tests, and when a test fails, it suggests ways to complete the model to make it pass the test.

Among the previous model completion approaches, four can also be applied to meta-models. DoMoRe works on domain models, like UML class diagrams and entityrelationship diagrams, and therefore can be used to add concepts of a domain of interest to meta-models. The approach of Kögel et al. [69] recommends complementary changes to a user editing action, and can be applied at the meta-model level, e.g. to recommend generalisation relations to a core super-class. Refacola is a refactoring constraint language and framework, extended to (meta-)model assistance in [130]. It is meta-level independent, providing assistance for completing partial (meta-)models to become syntactically correct. Finally, the Hermes framework can be configured with recommendation strategies. It is applicable to models within the EMF ecosystem, and hence to meta-models as well. Regarding the completion of model transformations, CONVErT [10] synthesises transformation code starting from examples of source and target models and their correspondences. Correspondences are specified manually, but there is also a recommender of likely correspondences based on similarity heuristics such as the name, structure and neighbourhood of model elements. AXSM [52] is a mapping recommender integrated in a tool to build data transformations via declarative mappings, from which translators written in XSLT, Java or ATL can be synthesised. AXSM recommends potential mappings based on heuristics grounded on the data schemas and on prior user selections.
Create. Only two of the analysed works target the creation of artefacts, one for meta-models and the other for models. The first one is DSL-maps [103]. Given the requirements of a DSL expressed as a mind-map, DSL-maps recommends meta-modelling patterns addressing them. The designer can select patterns among the ranked suggestions, and the tool combines the patterns to synthesise an initial meta-model, which the designer can then refine. The second approach is a modelling assistant for use case diagrams called UCcheck [9]. This tool has a wizard to create new use case diagrams using an existing one as a reference, from which suitable actors and use cases are recommended.

Find. The analysed papers include approaches to query repositories and suggest relevant artefacts for models and meta-models, but not for transformations or code generators. Extremo [123-125] is an extensible toolindependent assistant that helps finding relevant information for models and meta-models out of heterogeneous data sources (e.g. ontologies, XML schemas, RDF data, meta-models), and the results are ranked according to their suitability for the user. The rest of approaches are specific for some kind of model: the RS of Cerqueira et al. [26] finds and recommends sequence diagrams that match the user preferences; Matikainen et al. [81] tackle the problem of recommending the state machine from a library that implements the best policy to control a robot; and SBPR $[63,64]$ recommends process models from a repository according to the user business profile in LinkedIn (e.g. skills, interests and current position).

Repair. Repair approaches have been proposed for all kinds of artefacts: models, meta-models, transformations and code generators.

Regarding model repair, most works aim to recommend fixes for inconsistencies found in a given model (i.e. violations of the model's meta-model cardinality or wellformedness constraints). These approaches differ either in the applied technique to compute and rank the repairs, or in the application domain. In particular, IntellEdit [93] ranks quick fix solutions to model inconsistency problems according to the least-change principle; PARMOREL [11,53] determines the model repair actions based on the user preferences and on the experience gained from repairing under different personalisation settings; the diagram predicate framework (DPF) [108] and the approach by Nassar et al. [91] implement repairs as transformation rules; DIAGEN [82] represents models as hypergraphs and uses hypergraph patches to produce recommendations for repairing models; Refacola [130] uses constraint-based rules; BPMoQualAssess [60] provides guidelines to improve the actual value of quality 
metrics for business process models; B-repair [24] is specific to the B formal specification language and ranks the suggested repairs based on their estimated quality; Revision [98] tracks model inconsistencies to the editing action originating them in the model history and fixes this action to obtain a consistent model; MDSafeCer [87] detects missing information for supporting key evidence in process-based argumentations, and recommends how to resolve such deviations; ASIMOV [38] assists in the co-evolution of models and meta-models by proposing model co-evolution actions that a metamodeller must have defined previously; and Anguel et al. [8] also tackle the co-evolution problem, but they automatically fix resolvable changes and recommend coevolution actions to deal with non-resolvable changes.

There are also some model repair approaches that do not tackle model conformance, but they target other kinds of model-related problems. In particular, Mani et al. [79] compute repairs for input test models that make a code generator produce an incorrect output; in addition to complete, the suggestions for fixing behavioural tests in SMART [47] can also be classified as repairs; the Business Application Modeller (BAM) [137] permits specifying temporal rules for process models and, for some types of rules, it recommends how to fix their violations; and AMOR [21] is a model repository for model versioning that includes a recommender of possible resolutions for model conflicts.

With respect to meta-model repair, two of the works target OCL integrity constraints [12,28]. Batot et al. [12] tackle the co-evolution of OCL constraints upon metamodel changes. Their approach recommends a ranked list of OCL modifications that are correctly typed by the new meta-model version and minimise the number of changes and information loss. Clarisó et al. [28] repair OCL constraints which are too restrictive or too lax. Their method suggests weaker or stronger candidate versions of the problematic constraint, and the user can select one of them. In addition, two of the model repair approaches can be used to repair meta-models as well. PARMOREL allows repairing meta-models having duplicate attributes in related classes, or properties modelled both as attributes and as references [11]. Refacola [130], on the other hand, can help repairing syntactically incorrect meta-models, e.g. with inconsistent opposite or containment references (typical problems at the model level that can also happen in meta-models).

We found only one work supporting model transformation repair. This is anATLyzer [117-119], a tool integrated with the ATL IDE that identifies errors and recommends a ranked list of quick fixes to repair the transformation syntactically. Fixes are ranked taking into account the number of problems they solve, remaining errors and newly introduced errors.

Finally, we classify the approach by Mani et al. [79] as applicable to code generators because even if it suggests model repairs, these are applied in the context of code generation with XSLT.

Reuse. Recommenders in MDE have been applied to the reuse of models and transformations. Regarding model reuse, SimVMA [132] recommends Simulink models similar to the one that is being developed, and which the designer can import or clone for their reuse; REBUILDER [42] finds UML diagrams similar to a given query, and supports their full or partial composition into the given design; Paydar et al. [100,101] propose a reuse technique whereby the designer provides an input UML use case diagram, the most similar use cases are retrieved from a model repository, and then the activity diagrams associated to these use cases are semiautomatically adapted to (i.e. reused in) the new usage context; Koschmider et al. [50,51,71] propose both a recommender of process model fragments, and an explicit search facility to retrieve complete process models or fragments and insert them in the current modelling context, adapting them if needed; and Hermes [34-36] can incorporate model search strategies to find model elements suitable for reuse. Being generic, Hermes can also be applied to meta-models.

As for transformation reuse, it is supported by Refactory [111]. This tool permits defining generic refactorings over role models, so that developers can reuse the refactorings on new languages by binding the role model elements into elements of the language meta-model. Refactory includes a recommender that helps in identifying possible bindings, likely starting from some manually bound elements to avoid a high number of suggestions.

Other purpose. The remaining papers have very specialised purposes. Bobek et al. [17] propose a recommender for process modelling, which suggests the elements of a configurable diagram (i.e. a process with variability) that should be included in the current modelled process. MAGNET [16] guides users on the next tutorials to speed up the learning curve of a modelling tool. Finally, ModBud [116] is an envisioned framework to build assistants that educate novice modellers on abstraction. Such assistants may provide recommendations on a constructed model by comparison with a prescriptive model devised by the assistant.

In Table 3, the language-independent approaches have an asterisk $(*)$. We can see that most works are tied to a particular language, but a substantial amount of those applicable to models are language-independent. For this purpose, they 
are frequently defined over a meta-modelling frameworklike the Eclipse Modeling Framework (EMF) [131], Kermeta [57], GME [74], or DPF [109] — which enables their application to models of any language defined within the framework. This is the case of $[11,34-36,53,68,69,86,91,93,98,99,108$, 123-127,130]. In the case of DIAGEN [82], language independence is achieved by representing models as hypergraphs, and language definitions as hypergraph grammars. Other approaches are meta-level independent, and since metamodels are also models, such approaches are suitable for both meta-models and models $[5,6,11,34-36,53,68,69,123-125$, 130]; however, when applied to meta-models, the approaches are dependent on the meta-modelling language used, like EMF's Ecore. Finally, language independence can be a gradual term. For example, DoMoRe is language-independent as it is applicable to arbitrary domain models, but it cannot deal with other types of models such as behavioural models.

Table 4 summarises the languages that the languagedependent approaches handle. Most are widely used languages, like UML diagrams (11 approaches), business process models ( 9 approaches), Ecore (7 approaches), Simulink (2 approaches), OCL (2 approaches), XSLT (1 approach) and ATL (1 approach). The rationale is that building a RS generally involves a high effort and may require from training data, which may pay off for widespread languages, but the development may be too expensive for lesser used domainspecific languages (DSLs). There are some exceptions of RSs for DSLs though, typically embedded in tools built by the researchers $[10,52,92,111]$.

\subsection{Tooling}

Next, we analyse the tool support of the approaches using the criteria shown in the feature model of Fig. 6.

First, we look at the maturity of the supporting tools. We distinguish between proposals with no implementation, prototypes built as proof-of-concepts of the proposed ideas, and mature tools that make a full implementation available either as a framework, a plugin or a system. Frameworks typically offer generic functionality that can be customised by manually written code (e.g. by subclassing). Plugins encapsulate functionality that complements other tools, such as the Eclipse IDE. Systems can be either complete new applications that incorporate recommendation facilities or extensions of existing MDE tools with a RS.

Second, we classify the approaches as tool-independent if they can complement or be integrated into other MDE tools. The constraint in the feature model states that systems cannot be tool-independent, since the RSs are embedded in the tools themselves.

Third, RSs may trigger recommendations on demand, proactively, or both. In the first case, the user needs to explicitly start the recommendation process. In the latter case, the
Table 4 Languages targeted by recommender systems

\begin{tabular}{|c|c|c|}
\hline Language & Approach & Purpose \\
\hline \multicolumn{3}{|l|}{ Meta-Model } \\
\hline Ecore & $\begin{array}{l}\text { DoMoRe }[5,6] \\
\text { DSL-maps [103] } \\
\text { Extremo [123-125] } \\
\text { Hermes [34-36] } \\
\text { Kögel et al. [68,69] } \\
\text { PARMOREL }[11,53] \\
\text { Refacola [130] }\end{array}$ & $\begin{array}{l}\text { Complete } \\
\text { Create } \\
\text { Find } \\
\text { Repair } \\
\text { Reuse }\end{array}$ \\
\hline OCL & $\begin{array}{l}\text { Batot et al. [12] } \\
\text { Clarisó et al. [28] }\end{array}$ & Repair \\
\hline \multicolumn{3}{|l|}{ Model } \\
\hline Business process models & $\begin{array}{l}\text { BAM [137] } \\
\text { Bobek et al. [17] } \\
\text { BPMoQualAssess [60] } \\
\text { Deng et al. [31] } \\
\text { Koschmider et al. [50,51,71] } \\
\text { Li et al. [75] } \\
\text { MDSafeCer [87] } \\
\text { Rangiha et al. [110] } \\
\text { SBPR [63,64] }\end{array}$ & $\begin{array}{l}\text { Complete } \\
\text { Find } \\
\text { Repair } \\
\text { Reuse } \\
\text { Other }\end{array}$ \\
\hline DSL for embedded systems & DIG MDE [92] & Complete \\
\hline Mashup models & Baya $[27]$ & Complete \\
\hline Simulink & $\begin{array}{l}\text { Heinemann [49] } \\
\text { SimVMA [132] }\end{array}$ & $\begin{array}{l}\text { Complete } \\
\text { Reuse }\end{array}$ \\
\hline State/abstract machines & \begin{tabular}{|l|} 
B-repair [24] \\
Matikainen et al. [81]
\end{tabular} & \begin{tabular}{|l|} 
Find \\
Repair \\
\end{tabular} \\
\hline UML behavioural diagrams & SMART [47] & $\begin{array}{l}\text { Complete } \\
\text { Repair }\end{array}$ \\
\hline $\begin{array}{l}\text { UML class/ } \\
\text { structural diagrams }\end{array}$ & $\begin{array}{l}\text { AMOR [21] } \\
\text { Elkamel et al. [37] } \\
\text { IPSE [40] } \\
\text { ModBud [116] } \\
\text { RapMOD [72, 73] } \\
\text { REBUILDER [42] } \\
\text { Savary-Leblanc [121] }\end{array}$ & $\begin{array}{l}\text { Complete } \\
\text { Reuse } \\
\text { Other }\end{array}$ \\
\hline UML sequence diagrams & Cerqueira et al. [26] & Find \\
\hline UML use case diagrams & $\begin{array}{l}\text { Paydar et al. }[100,101] \\
\text { UCcheck [9] }\end{array}$ & $\begin{array}{l}\text { Create } \\
\text { Reuse }\end{array}$ \\
\hline \multicolumn{3}{|l|}{ Transformation } \\
\hline ATL & anATLyzer [117-119] & Repair \\
\hline DSL for refactorings & Refactory [111] & Reuse \\
\hline Marama Torua & AXSM [52] & Complete \\
\hline CONVErT & CONVErT [10] & Complete \\
\hline \multicolumn{3}{|l|}{ Code Generator } \\
\hline XSLT & Mani et al. [79] & Repair \\
\hline
\end{tabular}

RS makes recommendations without user intervention, when certain conditions are met.

Finally, we analyse the support for enacting the recommendations. This can be manual if the RS provides a list of recommendations and it is up to the user to decide how to use them; interactive if the RS permits the user to select a recommendation, which then becomes applied to the given context; automated if the recommendation is automatically applied without user intervention; and semiautomated if the recommendation enactment is automated, but the user may be prompted during the process, e.g. to input some value or decide between alternative options.

Table 5 classifies the revised papers according to these features. In the following, we discuss the different approaches attending to their maturity level, tool independence, recommender trigger, and recommendation enactment.

Maturity. The second column of Table 5 displays the maturity level of the approaches. When there are several 
Table 5 Recommender systems for MDE: tooling

\begin{tabular}{|c|c|c|c|c|c|c|c|c|}
\hline Approaches & Maturity & \begin{tabular}{|c|} 
Tool \\
Independent
\end{tabular} & $\begin{array}{l}\text { Recommend } \\
\text { OnDemand }\end{array}$ & $\begin{array}{l}\text { ler Trigger } \\
\text { Proactive }\end{array}$ & $\begin{array}{l}\text { Recomn } \\
\text { Manual }\end{array}$ & $\begin{array}{l}\text { mendation } \\
\text { l|Interact.| }\end{array}$ & $\begin{array}{l}\text { n Enac } \\
\text { Auto. }\end{array}$ & Semi- \\
\hline AMOR [21] & Plug-in & & $\checkmark$ & & & $\checkmark$ & & \\
\hline anATLyzer [117-119] & System & & $\checkmark$ & & & $\checkmark$ & & \\
\hline Anguel et al. [8] & Prototype & & $\checkmark$ & & & & & $\checkmark$ \\
\hline ASIMOV [38] & System & & $\checkmark$ & & & & & $\checkmark$ \\
\hline AXSM [52] & Plug-in & & $\checkmark$ & & & $\checkmark$ & & \\
\hline BAM [137] & Plug-in & & & $\checkmark$ & $\checkmark$ & & & \\
\hline Batot et al. [12] & Framework & $\checkmark$ & $\checkmark$ & & $\checkmark$ & & & \\
\hline Baya [27] & Prototype & & & $\checkmark$ & & $\checkmark$ & & \\
\hline Bobek et al. [17] & Prototype & & $\checkmark$ & & & & $\checkmark$ & \\
\hline BPMoQualAssess [60] & Prototype & & $\checkmark$ & & $\checkmark$ & & & \\
\hline B-repair [24] & System & & $\checkmark$ & & & $\checkmark$ & & \\
\hline Cerqueira et al. [26] & Prototype & & $\checkmark$ & & $\checkmark$ & & & \\
\hline Clarisó et al. [28] & Proposal & n.a. & $\checkmark$ & & $\checkmark$ & & & \\
\hline CONVErT [10] & Prototype & & & $\checkmark$ & & $\checkmark$ & & \\
\hline Deng et al. [31] & Prototype & & $\checkmark$ & & & $\checkmark$ & & \\
\hline DIAGEN [82] & System & & $\checkmark$ & & & $\checkmark$ & & \\
\hline DIG MDE [92] & System & & $\checkmark$ & & $\checkmark$ & & $\checkmark$ & \\
\hline DoMoRe $[5,6]$ & System & & $\checkmark$ & $\checkmark$ & & $\checkmark$ & & \\
\hline DPF [108] & Prototype & & $\checkmark$ & & & & $\checkmark$ & \\
\hline DSL-maps [103] & Plug-in & & $\checkmark$ & & & $\checkmark$ & & \\
\hline Elkamel et al. [37] & Prototype & & & $\checkmark$ & & $\checkmark$ & & \\
\hline Extremo [123-125] & Plug-in & $\checkmark$ & $\checkmark$ & & & $\checkmark$ & & \\
\hline Heinemann [49] & Prototype & & $\checkmark$ & & $\checkmark$ & & & \\
\hline Hermes [34-36] & Framework, Plug-in & $\checkmark$ & $\checkmark$ & $\checkmark$ & $\checkmark$ & $\checkmark$ & $\checkmark$ & $\checkmark$ \\
\hline IntellEdit [93] & Framework & & $\checkmark$ & & & $\checkmark$ & & \\
\hline IPSE [40] & Plug-in & & $\checkmark$ & & & $\checkmark$ & & \\
\hline Kermeta $[86]$ & Plug-in & & $\checkmark$ & & & & $\checkmark$ & \\
\hline Kögel et al. $[68,69]$ & Prototype & & & $\checkmark$ & $\checkmark$ & & & \\
\hline Koschmider et al. $[50,51,71]$ & Prototype & & $\checkmark$ & & & $\checkmark$ & & \\
\hline Li et al. $[75]$ & Prototype & & $\checkmark$ & & & $\checkmark$ & & \\
\hline MAGNET [16] & System & & $\checkmark$ & & $\checkmark$ & & & \\
\hline Mani et al. [79] & Prototype & & $\checkmark$ & & $\checkmark$ & & & \\
\hline Matikainen et al. [81] & Prototype & & & $\checkmark$ & & & $\checkmark$ & \\
\hline MDSafeCer [87] & Plug-in & & $\checkmark$ & & $\checkmark$ & & & \\
\hline ModBud [116] & Proposal & n.a. & $\checkmark$ & $\checkmark$ & $\checkmark$ & & & \\
\hline Nassar et al. [91] & Plug-in & & $\checkmark$ & & & $\checkmark$ & $\checkmark$ & \\
\hline PARMOREL $[11,53]$ & Plug-in & & $\checkmark$ & & & & $\checkmark$ & \\
\hline Paydar et al. $[100,101]$ & Prototype & & $\checkmark$ & & & $\checkmark$ & & \\
\hline PME [99] & Plug-in & & & $\checkmark$ & & & & $\checkmark$ \\
\hline Rangiha et al. [110] & Prototype & & & $\checkmark$ & & $\checkmark$ & & \\
\hline RapMOD $[72,73]$ & Plug-in & & & $\checkmark$ & & $\checkmark$ & & \\
\hline REBUILDER [42] & System & & $\checkmark$ & & $\checkmark$ & & & \\
\hline Refacola [130] & Prototype & $\checkmark$ & $\checkmark$ & & & $\checkmark$ & & \\
\hline Refactory [111] & Prototype & & $\checkmark$ & & $\checkmark$ & & & \\
\hline ReVision [98] & System & & $\checkmark$ & & & $\checkmark$ & & \\
\hline Savary-Leblanc [121] & Proposal & n.a. & unknown & unknown & & $\checkmark$ & & \\
\hline SBPR $[63,64]$ & System & & $\checkmark$ & & $\checkmark$ & & & \\
\hline Sen et al. $[126,127]$ & System & & $\checkmark$ & & $\checkmark$ & & & \\
\hline SimVMA [132] & Proposal & n.a. & $\checkmark$ & & & $\checkmark$ & & \\
\hline SMART [47] & System & & $\checkmark$ & & & $\checkmark$ & & \\
\hline UCcheck [9] & System & & $\checkmark$ & & & $\checkmark$ & & \\
\hline
\end{tabular}

We use NA as abbreviation for not applicable

incremental papers on the same approach, the column only shows the maturity achieved in the latest one (i.e. the highest maturity level). Among the 51 approaches, $4(7.8 \%)$ are proposals with no implementation, 19 $(37.2 \%)$ present prototypes as proof-of-concept, and the remaining $28(55 \%)$ provide full implementations. Most full implementations are either plug-ins (13) or systems/extensions of systems (13), while frameworks (3) are less pervasive. We categorise the Hermes [34-36] framework for developing RSs as a plug-in as well, as it uses a plug-in architecture that exposes and profits from Eclipse extension points.

Tool independence. This feature applies to approaches that make a prototype or full implementation available, but not to proposals that have not been realised in practice 


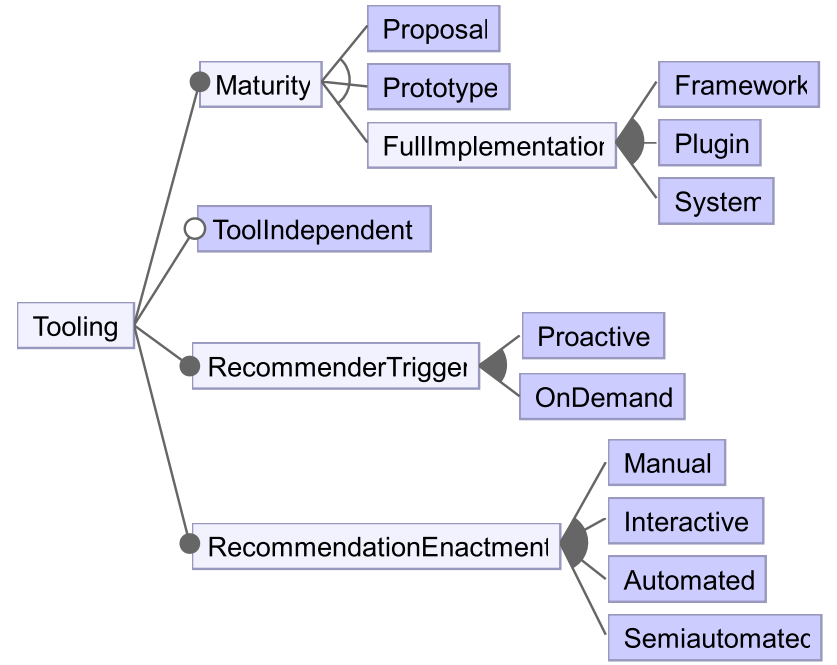

System $\Rightarrow \neg$ Toollndependent

Fig. 6 Tooling dimensions for RSs in MDE

(even though they may have the potential to become tool-independent). Most tool-supported RSs in MDE have been developed either as full software systems or as extensions of the following existing systems: the ATL development environment [117-119], some data mashup tools [27], the Generic Eclipse Modeling System (GEMS) [92], the Ecore Diagram Editor [5,6], DPF [108], DiaGen [82], Fujaba [40], AutoFOCUS3 [16], the AMASS platform [87], the AMOR model versioning system [21], Kermeta [86], the Generic Modeling Environment (GME) [99], Sparx Enterprise Architect [72,73] and the meta-modelling tool $\mathrm{AToM}^{3}$ [126,127]. All these approaches built as complete systems or system extensions are tool-dependent (84.31\%). In some cases, the tools are implemented atop EMF to achieve generality. However, we only consider that an approach is toolindependent if, in addition, it provides explicit means to facilitate its integration with other tools. Under this perspective, only four (7.84\%) approaches are truly independent from any modelling tool. We comment on these approaches next.

The framework developed by Batot et al. [12] recommends how to co-evolve OCL invariants upon Ecore meta-model changes (i.e. it is language-dependent); however, the framework is not specific for particular editors, and is extensible with new heuristics to guide the search of recommendations. Refacola [130] achieves tool independence by being based on a constraint-based domainspecific language to specify model-assistance operations. Extremo [123-125] is a modelling assistant that defines extension points (the extensibility mechanism provided by Eclipse) to allow its integration with external modelling and meta-modelling tools within Eclipse. Finally,
Hermes [34-36] is not a concrete RS but a framework with a plugin-based architecture to develop RSs within Eclipse. Its extension points allow defining new recommendation strategies and the integration with modelling editors and heterogeneous data repositories.

Other approaches can be used with several tools, but are still tool-dependent. This is the case of UCcheck [9], an assistant for use case diagrams coded in Python that supports use case diagrams specified with TTool-a free software from Telecom Paris-and the Cameo Systems Modeler.

Recommender trigger. As the fourth column of Table 5 shows, most RSs provide recommendations on user demand (41 approaches out of 51, an 80.39\%). Fewer approaches provide recommendations proactively without user intervention (12 out of 51, a $23.53 \%$ ), typically by monitoring the user editing actions to update the recommendations in return. Only a few tools ( 3 of them, a $5.88 \%$ ) can trigger the recommendations both on demand and proactively: the recommender of domain model elements DoMoRe [5,6], the envisioned modelling learning environment ModBud [116] and the generic RS framework Hermes [34-36]. Finally, Savary-Leblanc [121] does not give enough details on how to access the recommendations, so we mark it as unknown in the table.

Recommendation enactment. The last four columns in Table 5 display how the works enact the recommendations. In most cases, recommendations can be applied either manually $(31.37 \%)$ or interactively $(49.02 \%)$. Automated enactments typically occur in model completion and model repair. As an example, DIG MDE [92] automatically completes a model, and if this is not possible, it recommends the user how to fix the model manually. In turn, the tool by Nassar et al. [91] permits repairing models either automatically or interactively. Three approaches $(5.88 \%)$ provide semiautomated enactment of recommendations: two are co-evolution approaches $[8,38]$ that automatically infer and apply a migration strategy, but the user may need to select between alternative solution steps, e.g. in the case of nonresolvable changes; the other corresponds to the proactive modelling approach in PME [99], where models are automatically modified according to the models' meta-model, and the user is only prompted if several optional modifications exist. Finally, since Hermes [34-36] is a framework to build RSs, it provides mechanisms to support all types of recommendation enactment.

\subsection{Recommendation}

MDE researchers have applied diverse recommendation approaches for a variety of tasks and purposes. In this sec- 


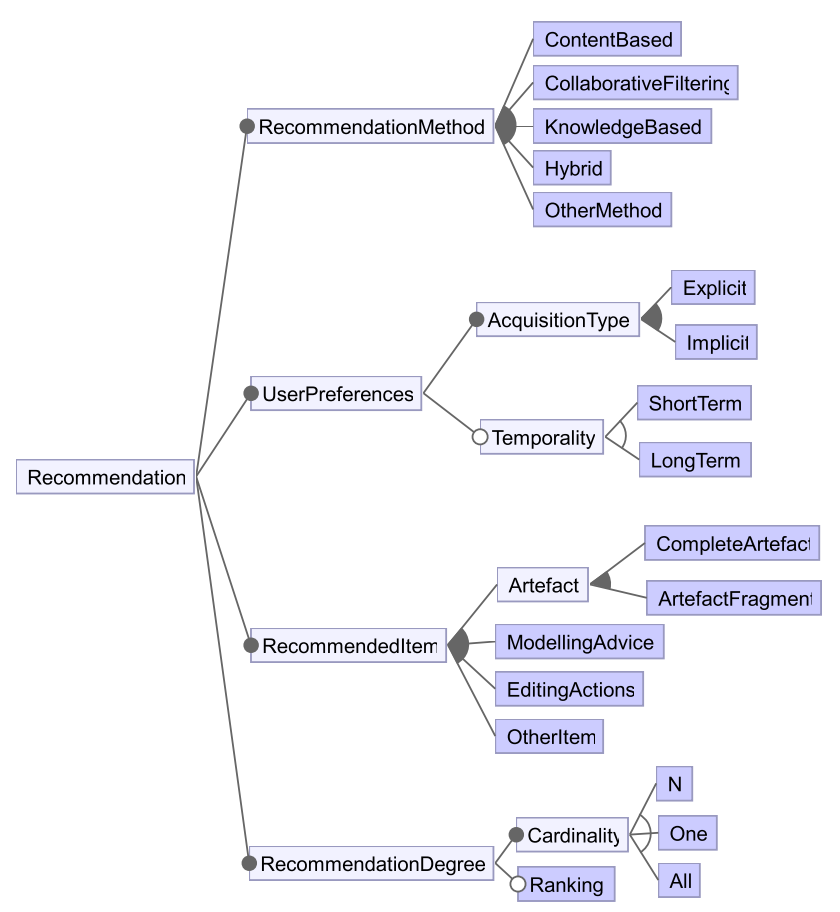

Fig. 7 Recommendation dimensions for RSs in MDE

tion, we characterise, categorise and analyse the works on MDE recommenders according to the features shown in the diagram of Fig. 7.

As a first feature of analysis, we consider the recommendation method used. The majority of the RSs apply one of the four main techniques explained in Sect. 2.2: contentbased, collaborative filtering, knowledge-based and hybrid. In addition, some works use ad-hoc techniques that do not fall into the previous categories. They are represented by OtherMethod in Fig. 7.

Second, RSs collect user information to provide personalised recommendations (feature UserPreferences in the diagram). In this respect, we investigate how this information is collected (feature AcquisitionType). In some cases, the user's preferences are gathered implicitly by monitoring the user interactions with the system or analysing the current state of the modelling/MDE activity. In other cases, the user needs to explicitly provide his/her preferences to the system, for example via questionnaires. In addition, we examine the temporality of the collected preferences, which can reflect recent, likely temporal preferences for the task at hand (i.e. ShortTerm) or more general and enduring preferences (i.e. LongTerm).

Third, we analyse the types of items provided as recommendations (feature RecommendedItem). These can be complete artefacts (e.g. a model), fragments of an artefact (e.g. a class), advices that the user can profit from during a modelling activity, or editing actions (e.g. in the context of model repair). The diagram includes the OtherItem feature for items that do not fall in any of the previous categories.

Finally, the feature RecommendationDegree comprises the amount of recommendations presented to the user (Cardinality) and whether they are ranked (Ranking).

Table 6 categorises the surveyed approaches according to these features. Taking this categorisation into consideration, we start by analysing the approaches attending to the recommendation method they use, and then, we analyse them based on the other features.

Content-based. These approaches use different content encoding and similarity notions to represent and relate items for generating personalised recommendations.

First, we comment on the content-based approaches that recommend complete artefacts. Cerqueira et al. [26] compare two alternative encodings of sequence diagrams (bag-of-words and a vector encoding structural features) for the recommendation of sequence diagrams matching the user's preferences. The RS uses a content-based filtering algorithm to find the closest sequence diagrams. The RS proposed by Paydar et al. [100,101] facilitates the reuse of models with functional requirements of web applications. For this purpose, the system recommends similar use cases to the one provided by the user and then adapts the activity diagrams linked to the selected use case to the provided one. Item similarities are computed based on name similarity of the use case elements and on the diagram context. SimVMA [132] uses clone detectors to estimate similarities. It uses nearmiss clones to recommend similar Simulink models, from which low-granularity recommendations can also be extracted.

Similarity has also been exploited to recommend artefact fragments. For example, Elkamel et al. [37] use similarity metrics to suggest similar classes to newly created classes. The developer may accept the suggested classes with all or some of their attributes and methods. DoMoRe $[5,6]$ addresses the same problem by means of semantic similarities. It relies on an extensive knowledge database — called SemNet —of several million domain-specific terms and their relationships to provide context-sensitive recommendations. In particular, DoMoRe suggests names for new elements, and possible related concepts to the selected one (e.g. upon selecting a class, the system suggests possible sub-/superclasses, container and aggregated classes, related and associated classes). Savary-Leblanc [121] envisions a RS that calculates the similarity using semantic distances obtained from lexical databases like WordNet [84]. Extremo [123125] also employs semantic similarity based on WordNet to provide a ranked list of recommended model elements, upon an explicit query of the user. 
Table 6 Recommender systems for MDE: recommendation method

\begin{tabular}{|c|c|c|c|c|c|}
\hline \multirow[b]{2}{*}{ Approaches } & \multirow[b]{2}{*}{ Acquisition Type } & \multirow[b]{2}{*}{ Temporality } & \multirow[b]{2}{*}{ Recommended Item } & \multicolumn{2}{|c|}{ Recommendation Degree } \\
\hline & & & & Cardinality & Ranking \\
\hline \multicolumn{6}{|l|}{ Content-based } \\
\hline AXSM [52] & Implicit, Explicit & Short-term & Artefact fragment & All & $\checkmark$ \\
\hline Cerqueira et al. [26] & Implicit, Explicit & Short-term & Complete artefact & $\mathrm{N}$ & $\checkmark$ \\
\hline CONVErT [10] & Implicit, Explicit & Short-term & Artefact fragment & $\mathrm{N}$ & $\checkmark$ \\
\hline DoMoRe $[5,6]$ & Implicit & Short-term & Artefact fragment & All & $\checkmark$ \\
\hline Elkamel et al. [37] & Implicit & Short-term & Artefact fragment & All & \\
\hline Extremo [123-125] & Explicit & Short-term & Artefact fragment & All & $\checkmark$ \\
\hline Paydar et al. $[100,101]$ & Implicit & Short-term & Complete artefact & $\mathrm{N}$ & $\checkmark$ \\
\hline Refactory [111] & Implicit & Short-term & Artefact fragment & $\mathrm{N}$ & \\
\hline Savary-Leblanc [121] & Implicit & unknown & Artefact fragment & unknown & \\
\hline SimVMA [132] & Implicit, Explicit & Short-term & Complete artefact, artefact fragment & All & $\checkmark$ \\
\hline \multicolumn{6}{|l|}{ Collaborative filtering } \\
\hline MAGNET [16] & Implicit & Short-term & Modelling advice & All & $\checkmark$ \\
\hline Matikainen et al. [81] & Implicit & Short-term & Complete artefact & $\mathrm{N}$ & $\checkmark$ \\
\hline ModBud [116] & Implicit, Explicit & unknown & Modelling advice & unknown & unknown \\
\hline PARMOREL $[11,53]$ & Implicit, Explicit & Long-term & Editing actions & One & \\
\hline \multicolumn{6}{|l|}{ Knowledge-based } \\
\hline AMOR [21] & Implicit & Short-term & Editing actions & All & $\checkmark$ \\
\hline Anguel et al. [8] & Implicit & Short-term & Editing actions & All & \\
\hline ASIMOV [38] & Implicit & Short-term & Editing actions & $\mathrm{N}$ & \\
\hline BAM [137] & Implicit & Short-term & Editing actions & All & $\checkmark$ \\
\hline Baya $[27]$ & Implicit & Short-term & Artefact fragment & $\mathrm{N}$ & $\checkmark$ \\
\hline Bobek et al. [17] & Implicit & Short-term & Artefact fragment & $\mathrm{N}$ & $\checkmark$ \\
\hline BPMoQualAssess [60] & Implicit & Short-term & Modelling advice & All & \\
\hline Deng et al. [31] & Implicit & Short-term & Artefact fragment & $\mathrm{N}$ & $\checkmark$ \\
\hline DIAGEN [92] & Implicit & Short-term & Editing actions & All & \\
\hline DIG MDE [92] & Implicit, Explicit & Short-term & Artefact fragment & All & \\
\hline DPF [108] & Implicit & Short-term & Artefact fragment & All & \\
\hline DSL-maps [103] & Implicit & Short-term & Artefact fragment & All & $\checkmark$ \\
\hline IPSE [40] & Implicit & Short-term & Artefact fragment, modelling advice & One & \\
\hline Kermeta [86] & Implicit & Short-term & Artefact fragment & All & \\
\hline Li et al. [75] & Implicit & Short-term & Artefact fragment & $\mathrm{N}$ & $\checkmark$ \\
\hline Mani et al. [79] & Implicit & Short-term & Editing actions & All & \\
\hline MDSafeCer [87] & Implicit & Short-term & Modelling advice & All & \\
\hline Nassar et al. [91] & Implicit & Short-term & Editing actions & All & \\
\hline RapMOD $[72,73]$ & Implicit & Short-term & Artefact fragment & $\mathrm{N}$ & $\checkmark$ \\
\hline REBUILDER [42] & Implicit & Short-term & Complete artefact, artefact fragment & All & $\checkmark$ \\
\hline Refacola [130] & Implicit & Short-term & Editing actions & $\mathrm{N}$ & \\
\hline ReVision [98] & Implicit & Short-term & Editing actions & All & $\checkmark$ \\
\hline Sen et al. $[126,127]$ & Implicit, Explicit & Short-term & Artefact fragment & $\mathrm{N}$ & \\
\hline UCcheck [9] & Implicit & Short-term & Modelling advice & All & \\
\hline \multicolumn{6}{|c|}{ Hybrid: Content-based, collaborative filtering } \\
\hline Heinemann [49] & Implicit & Short-term & Artefact fragment & $\mathrm{N}$ & $\checkmark$ \\
\hline Kögel et al. $[68,69]$ & Implicit & Short-term & Editing actions & $\mathrm{N}$ & \\
\hline Koschmider et al. $[50,51,71]$ & Implicit, Explicit & Short-term & Complete artefact, artefact fragment & $\mathrm{N}$ & $\checkmark$ \\
\hline \multicolumn{6}{|c|}{ Hybrid: Content-based, knowledge-based } \\
\hline B-repair [24] & Implicit & Short-term & Editing actions & $\mathrm{N}$ & $\checkmark$ \\
\hline \multicolumn{6}{|c|}{ Hybrid: Content-based, social-based } \\
\hline Rangiha et al. [110] & Implicit, Explicit & Long-term & Artefact fragment & All & $\checkmark$ \\
\hline SBPR $[63,64]$ & Implicit & Short-term & Complete artefact & All & $\checkmark$ \\
\hline \multicolumn{6}{|l|}{ Other Method } \\
\hline anATLyzer [117-119] & Implicit & Short-term & Editing actions & All & $\checkmark$ \\
\hline Batot et al. [12] & Implicit & Short-term & Editing actions & $\mathrm{N}$ & $\checkmark$ \\
\hline Clarisó et al. [28] & Implicit, Explicit & Short-term & Editing actions & $\mathrm{N}$ & $\checkmark$ \\
\hline IntellEdit [93] & Implicit & Short-term & Editing actions & All & $\checkmark$ \\
\hline PME [99] & Implicit & Short-term & Artefact fragment & All & $\checkmark$ \\
\hline SMART [47] & Implicit & Short-term & Editing actions & All & \\
\hline \multicolumn{6}{|l|}{ Any Method } \\
\hline Hermes [34-36] & Implicit, Explicit & Long-term & Editing actions & $\mathrm{N}$ & $\checkmark$ \\
\hline
\end{tabular}


Content-based similarity has been applied to transformation development as well. CONVErT [10] helps discovering and specifying transformation correspondences using concrete visualisations. A RS suggests mappings between source and target models based on different similarity heuristics, choosing mappings that resemble examples provided by the user. In a similar vein, AXSM [52] recommends mappings based on similarity criteria (source/target element tag names, element types, structural similarity, example data item equivalences) and previous user selections within the Marama Torua tool. Refactory [111] supports the definition of generic refactorings over role models so that developers can reuse the refactorings on new languages by mapping the role model elements into elements of the language meta-model. The tool includes a RS to complete the mapping using structural similarity and other heuristics, like name similarity.

Collaborative filtering. These approaches exploit information about past behaviour or opinions from the user community [56]. In some cases, users correspond to developers for which personalised recommendations are generated, and in other cases, users (and items) are mapped to elements within the artefacts that are target of recommendations.

MAGNET [16], PARMOREL [11,53] and ModBud [116] belong to the first case. MAGNET is a RS within the AutoFOCUS3 modelling tool to help beginners to learn using the tool. It monitors user actions and proposes short videos illustrating what to do next. The RS model is based on data collected during a tutorial with a previous set of users. PARMOREL uses reinforcement learning to find a sequence of actions that repairs the issues present in a model. The algorithm initially reuses the experience obtained from other users' repairs and learns after each repair. ModBud is an envisioned framework to build modelling bots to assist novice users. The authors foresee using machine learning to predict good modelling decisions for given design requirements.

Matikainen et al. [81] address the second case. Their RS selects the best-performing state machine to control a robotic vacuum cleaner. Room layouts are interpreted as users, robot state machines as items, and item ratings are based on the performance of the robot state machines on the room layouts.

Knowledge-based. Most approaches belong to this category. They use techniques that can be generally classified as constraint-based or case-based. Constraint-based techniques determine the recommendations by looking for a set of items that fulfil established domain-dependent rules. Case-based techniques, in contrast, provide recommendations to a problem by examining past solutions for alike problems (cases) [56].
Some of the constraint-based recommenders found in the literature are built upon technologies such as Alloy and Prolog. Specifically, Sen et al. [126] use Prolog as a backend of the $\mathrm{AToM}^{3}$ language workbench [29] to suggest completions of a partial model. The work was extended by using Alloy [127] to recommend the closest valid complete model within a given scope. Kermeta [86] also uses Alloy to provide completion suggestions. Refacola [130] provides a constraint-based language to express modelassistance operations in a declarative way. In the domain of education, IPSE [40] relies on Prolog to guide users in creating a class diagram. The guidelines are explicitly modelled by the teacher by means of constraints suggesting hints whenever matched. For the domain of embedded systems, DIG MDE [92] uses Prolog to guide the user in completing combinatorially challenging modelling problems on the basis of user-defined rules.

RSs for completion and repair are sometimes based on (graph transformation) rules. DPF [108] computes completion rules which ensure the satisfaction of wellformedness predicates. RapMOD [72] matches editing operations in UML structural diagrams to a catalogue of modelling activities and ranks the candidate activities by relevance. Different from model completion, rule-based model repair may require deleting elements to obtain a valid model. Hence, Nassar et al. [91] derive graph transformation programs able to fix an invalid model by first deleting superfluous objects and links and then adding necessary elements. DIAGEN [82] uses hypergraph grammar rules and hypergraph patches (graph modifications) to propose both model completions and repairs. Similarly, ReVision [98] proposes model repairs based on consistency-preserving editing rules, with heuristics that avoid undoing former editing steps.

For quality assurance, BPMoQualAssess [60] recommends improvements for process models based on rules modelling expected quality criteria (e.g. regarding size, nesting levels and element ratios), and UCcheck [9] provides advices for improving use case diagrams based on sets of rules and guidelines.

Recommenders for model/meta-model co-evolution can also be rule-based. This is the case of ASIMOV [38], where the language designer specifies the migration assistance rules, and modellers use them to obtain recommendations for model migration. In contrast, the approach by Anguel et al. [8] suggests a migration strategy based on meta-model matching and the use of logic programming.

Some rule-based RSs target behavioural models. MDSafeCer [87] recommends how to resolve flaws of safety argumentations attached to process models. For this purpose, it first identifies the problematic elements, and then uses rules to provide advices to resolve the deviations. 
Also for process modelling, BAM [137] uses modelchecking to detect errors in process models, and suggests corrections for the errors in relation to user-defined validation rules and Dwyer's temporal specification patterns [33].

Some works use patterns following a case-based approach. In particular, Baya [27] relies on a knowledge base of curated patterns, several similarity metrics and ranking algorithms as a basis for the recommendation of the next steps when building mashup models. Moreover, it applies weaving to incorporate the recommended pattern into the mashup model. The process model recommenders of $\mathrm{Li}$ et al. [75] and Deng et al. [31] extract task relations and patterns from process models, which are then used to recommend activity nodes for the current model. The AMOR [21] model versioning system recommends resolution patterns for conflicts between two model versions. The patterns can be mined from repositories or specified manually. DSL-maps [103] uses a catalogue of patterns to transition from the requirements of a DSL (given as a mind-map) to its design (given as a meta-model). It performs a lexical analysis of the requirements to match them against an ontology-based description of the patterns, and suggests a ranked list of patterns to realise the requirements. Mani et al. [79] also use patterns to assist when repairing faults in input models of code generators. Their approach identifies correct output fragments that are similar to the incorrect one, and suggests repair actions based on run-time data.

Finally, probabilistic forms of knowledge representation are also possible. For example, REBUILDER [42] combines case-based reasoning with WordNet and Bayesian networks to enable reusing UML diagrams, or part of them. Bobek et al. [17] also use Bayesian networks to recommend following tasks when instantiating a configurable process model.

Hybrid. Some works combine several recommendation methods to benefit from their strengths and mitigate particular limitations. The surveyed papers have combined content-based techniques with collaborative filtering, social-based and knowledge-based methods.

Three approaches combine collaborative filtering with content-based recommendations. The first one, by Kögel et al. [68,69], recommends model changes by looking at the previous model history (e.g. what other developers did on previous model versions) and co-occurring model changes. Heinemann [49] evaluates the use of association rules and collaborative filtering to recommend Simulink library elements for the current model. The collaborative filtering method considers models as users and elements as items. Finally, the RS of Koschmider et al. [50,51,71] uses both similarity metrics and frequency of use by the community to recommend complete process models or fragments.

For behavioural modelling, B-repair [24] suggests automatic repairs of faulty models written in the B formal specification language. The approach uses two types of rules (hence being knowledge-based) to suggest fixes in state machine transitions. Then, it uses machine learning (features learnt from state machine transitions, a contentbased approach) to estimate the quality of the repairs and rank the recommendations.

Finally, SBPR $[63,64]$ combines the traditional contentbased approach with social-based recommendation to suggest business process models for reuse. For this purpose, it extracts information from the user profile in LinkedIn. ${ }^{2}$ Similarly, the approach by Rangiha et al. [110] profits from social tagging to recommend suitable actors and roles in a social business process modelling tool. In addition, it recommends tasks based on similarity metrics.

Other method. A few works use non-traditional recommendation methods based on search and static analysis. On the one hand, two approaches use model search as the underlying technique for recommendation, both targeting OCL. Clarisó et al. [28] generate potential fixes to OCL constraints by using mutation. Batot et al. [12] tackle the co-evolution of OCL constraints and meta-models using multi-objective optimisation guided by criteria like correctness and minimisation of changes and information loss.

On the other hand, several works provide recommendations out of the static analysis of models, meta-models or OCL expressions. PME [99], which extends the generic modelling environment (GME) to support proactive modelling, recommends further editing actions (e.g. connecting an object to another) upon user actions (e.g. selecting an object). The recommendations are created by the syntactic analysis of the meta-model and OCL constraints. IntellEdit [93] recommends quick fixes for repairing models based on the static analysis of failing OCL expressions. It ranks the recommended fixes by the amount of required changes (from lower to higher). AnATLyzer [117-119] extends the ATL IDE for developing model transformations with the detection of type errors and suggestions of quick fixes. Errors are detected by static analysis and model finders. The proposed quick fixes are ranked by the number of errors that they correct. The ranking can be calculated dynamically using speculative analysis [88] (i.e. the simulated execution of all possible repairs and the analysis of their consequences),

\footnotetext{
${ }^{2}$ https://www.linkedin.com/.
} 
or statically using rankings pre-computed on a set of transformations with injected faults. Finally, SMART [47] supports test-driven development of UML models. It statically analyses the test cases and their execution $\operatorname{logs}$ to report errors. Moreover, it suggests quick fixes for automatically solving structural errors (e.g. adding a missing model element) and provides guidance to solve behavioural errors triggered during the test case execution (e.g. displaying a sequence diagram with the test case execution, or a summary of the changes in attribute values or the model state).

Any method. The framework Hermes [34-36] for the creation of RSs can be extended with any recommendation strategy and recommendation method. It provides facilities to define the recommendation context, which can be obtained either implicitly or explicitly. Developers of RSs can persist user preferences (long-term temporality) and set filters and ranks for their strategies.

Once we have classified the works according to the recommendation method, we characterise how they collect the user's preferences (acquisition type), the temporality of those preferences and the size and ordering of the recommendation sets (recommendation degree).

Acquisition type. All works but Extremo collect data implicitly. The most common type of implicit data is the user's previous interaction with the system (including the current selection of elements in the editor) and the inprogress model. In some cases, like SBPR, this includes user information from LinkedIn.

In addition, 12 approaches $[10,11,26,28,50-53,71,92$, $110,116,123-127,132]$ also collect data explicitly. In these cases, data is acquired through questionnaires, parameters, tags or requirement definitions, in combination with implicit data acquisition methods like analysing the user's in-progress model.

Temporality. Most approaches (90\%) collect preferences for their use during a short period of time, typically the current modelling session or model state. PARMOREL $[11,53]$ uses long-term preferences by storing the experience gained from each repair, allowing the algorithm to improve its performance in consecutive executions. The process model recommender of Rangiha et al. [110] exploits persistent social tags to express, e.g. required skills for tasks and skill-sets of users. Hermes [34-36], being a framework, enables developers to persist user preferences as required by the recommendation strategy. Finally, two works $[116,121]$ do not provide detailed temporality information.

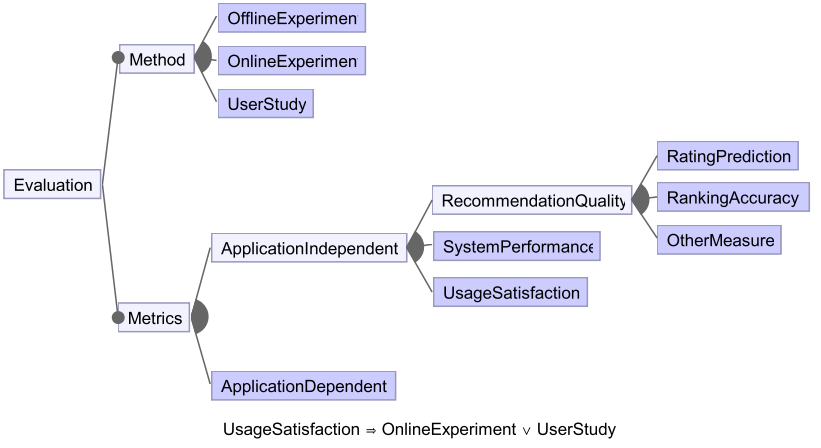

Fig. 8 Evaluation dimensions for RSs in MDE

Recommendation degree. When it comes to the recommended items, most approaches (53\%) present all recommendations found by the method to the user. Since this might be overwhelming if there are many options, some approaches (4\%) present just one recommendation, and others (39\%) present the top $N$ recommendations.

The filtering criteria vary depending on the recommendation method, and are frequently used to rank the suggestions. Examples of filtering criteria include the most similar models (as in [26]), the quick fixes repairing more errors (as in [119]), or the fixed model or constraint having the lowest number of modifications with respect to the original one (as in $[28,93]$ ). Sometimes, the quality of the recommendation is calculated using pre-trained machine-learning models, as in [24]. In the case of anATLyzer [119], the user can choose either a fast but less accurate ranking of recommended quick fixes (based on a pre-calculated estimation of the repair power of quick fixes); or a slower but more accurate one (based on a speculative application of the quick fixes to the current transformation). As Table 6 shows, some early works do not provide information about cardinality or ranking.

\subsection{Evaluation}

In this section, we review how the RSs have been evaluated on the basis of the two orthogonal features shown in Fig. 8: the followed evaluation methods and the used evaluation metrics.

As the figure shows, we first distinguish three types of evaluation methods [45]: offline experiments, online experiments, and user studies. Offline experiments correspond to analytical studies on datasets, online experiments are usercentric studies that evaluate the system in a real setting, and user studies consist of experiments planned for small groups of participants.

In addition, we identify several metrics to assess different recommendation goals and quality measures. We classify the metrics as application-independent and applicationdependent. In turn, application-independent metrics are 
divided into three groups. The first one comprises traditional metrics used to evaluate RSs regardless of the application or task for which they have been developed. Here, we distinguish between recommendation quality (accuracy) metricsi.e. rating prediction metrics (e.g. MAE and RMSE) and ranking accuracy metrics (e.g. precision, recall, nDCG and MRR) - and other measures that capture non-accuracy recommendation characteristics, such as diversity, coverage and novelty. A second group of domain-independent metrics is related to system performance, such as consumed time and required resources to perform a task. The third group of domain-independent metrics is formed by usage satisfaction metrics, such as user engagement, perceived usefulness and trust on the system, as well as system usability, responsiveness, security and privacy. As Fig. 8 shows, measuring usage satisfaction requires performing on-line experiments or user studies, as offline experiments do not involve users.

Finally, application-dependent metrics are devised for particular MDE applications and tasks. They include metrics such as the average number of constraint violations in model repair recommendations, or the total number of valid matches in the recommendation of model transformation mappings.

Table 7 categorises the analysed RSs according to the method (offline, online and user study) and metrics used for their evaluation. An approach can appear multiple times in the table if it was evaluated by means of several methods. Additionally, Table 8 presents a matrix crossing the methods and metrics used in the papers. Overall, we can observe that online experiments are the least used evaluation method, and that neither rating prediction nor non-accuracy metrics are used; the former are indeed in disuse in the RS research field. A total of 19 approaches (37\%) have no evaluation.

Offline experiment. This is the most popular evaluation method, used in 21 of the revised approaches. Making use of data records with past user behaviour and feedback, among other information, offline experiments simulate past and present real conditions without requiring the participation of users during the evaluation process.

These experiments exploit available datasets to compute a variety of aspects about a RS, such as its scalability and performance, the precision and quality of its recommendations, and the reduction of modelling effort. However, since data repositories of models are not as common as, e.g. those for programming languages, a fundamental issue about offline experimentation for MDE recommenders is the availability of artefacts over which the evaluation can be performed. To address this issue, we have observed four solutions in the literature. The first one is the generation of synthetic data. For the case of repair recommenders, the set of artefacts is typically generated by applying mutation operators over a set of seed artefacts to obtain faulty artefact variants. This approach was used by anATLyzer to evaluate the recommendation of quick fixes over transformations [117-119]; by B-repair to evaluate fixes over state machines [24]; by IntellEdit to evaluate if its content-assistant solves errors in models [93]; by Matikainen et al. [81] to evaluate the recommendation of state machines for robotic cleaners; and by Mani et al. [79] to evaluate the effectiveness of its model repair recommender. The seed artefacts may come from third parties (as in the case of anATLyzer and Mani et al.), be generated synthetically (as in IntellEdit and Matikainen et al.) or manually (as in B-repair). A second solution is to locate repository sources of the appropriate type. For example, CONVErT was evaluated through models from the Illinois Semantic Integration Archive [10], Extremo gathered heterogeneous information from several sources such as OMG meta-models or the AtlanMod meta-model Zoo [123], Refacola used the whole AtlanMod Ecore meta-model Zoo [130], Heinemann used models of a Simulink repository [49], Matikainen et al. used floor plans from the Google SketchUp database of 3D models [81], and the evaluation of SBPR involved process models from different sources [64]. A third solution consists of taking example artefacts from published papers (as in B-repair), or datasets used by other authors (as in the case of Kögel et al. in 2016 [68,69]). Finally, another solution is to obtain real-world artefacts from companies, like Li et al. [75] and Deng et al. [31], who used a dataset of 221 business processes collected from a local government in China, in combination with a synthetic dataset. Table 9 shows the public (i.e. available) datasets and repositories used in the surveyed papers.

In addition to mutating artefacts to introduce faults, we have found other modifications in artefacts. In RSs for model completion, the models of the considered dataset are removed elements to enable triggering the recommendations, and their effects are compared with the original model. This is the strategy followed by Heinemann [49], Li et al. [75], Deng et al. [31] and Baya [27]. In the first case, half of the model elements were removed; in the second case, the recommendation starts from the second activity node; and in the two last cases, model portions of increasing size were systematically removed.

Some approaches require training the recommender. For this purpose, the dataset is partitioned into sets for training and validation, as done by $\mathrm{Li}$ et al. [75], Deng et al. [31] and Heinemann [49]. To estimate the generalisability of the method and avoid problems related to overfitting and selection bias, k-fold cross-validation is recommended for statistical analysis [25]. This way, Deng et al. [31] use 5-fold cross-validation: the dataset is partitioned into five subsets, one is taken for validation (testing), the rest for training, and the procedure is 
Table 7 Recommender systems for MDE: evaluation

\begin{tabular}{|c|c|c|c|c|c|c|}
\hline Approaches & \begin{tabular}{|l} 
Rating \\
Prediction
\end{tabular} & $\begin{array}{r}\text { Appli } \\
\text { Ranking } \\
\text { Accuracy }\end{array}$ & $\begin{array}{l}\text { ication In } \\
\text { Other } \\
\text { Measure }\end{array}$ & $\begin{array}{l}\text { System } \\
\text { Performance }\end{array}$ & \begin{tabular}{|l} 
Usage \\
Satisfaction
\end{tabular} & $\begin{array}{l}\text { Application } \\
\text { Dependent }\end{array}$ \\
\hline \multicolumn{7}{|l|}{ Offline experiment } \\
\hline AMOR [21] & & & & & & $\checkmark$ \\
\hline anATLyzer [119] & & & & & & $\checkmark$ \\
\hline Batot et al. [12] & & & & & & $\checkmark$ \\
\hline Baya [27] & & $\checkmark$ & & $\checkmark$ & & \\
\hline B-repair [24] & & $\checkmark$ & & & & \\
\hline CONVErT [10] & & $\checkmark$ & & & & \\
\hline Deng et al. [31] & & $\checkmark$ & & $\checkmark$ & & \\
\hline DIAGEN [82] & & & & $\checkmark$ & & \\
\hline DIG MDE [92] & & & & $\checkmark$ & & \\
\hline Extremo [123-125] & & & & $\checkmark$ & & $\checkmark$ \\
\hline Heinemann [49] & & $\checkmark$ & & & & \\
\hline IntellEdit [93] & & $\checkmark$ & & & & $\checkmark$ \\
\hline Kögel et al. [68,69] & & $\checkmark$ & & & & \\
\hline Li et al. [75] & & $\checkmark$ & & $\checkmark$ & & \\
\hline Mani et al. [79] & & & & & & $\checkmark$ \\
\hline Matikainen et al. [81] & & & & & & $\checkmark$ \\
\hline PARMOREL $[11,53]$ & & & & $\checkmark$ & & \\
\hline PME [99] & & & & $\checkmark$ & & $\checkmark$ \\
\hline Refacola [130] & & $\checkmark$ & & $\checkmark$ & & $\checkmark$ \\
\hline Refactory [111] & & & & & & $\checkmark$ \\
\hline SBPR [64] & & $\checkmark$ & & & & \\
\hline \multicolumn{7}{|l|}{ Online experiment } \\
\hline ASIMOV [38] & & & & $\checkmark$ & & $\checkmark$ \\
\hline DoMoRe $[5,6]$ & & & & & $\checkmark$ & \\
\hline \multicolumn{7}{|l|}{ User study } \\
\hline anATLyzer [119] & & & & & & $\checkmark$ \\
\hline AXSM [52] & & & & & $\checkmark$ & \\
\hline Baya [27] & & & & $\checkmark$ & $\checkmark$ & \\
\hline Cerqueira et al. [26] & & $\checkmark$ & & & $\checkmark$ & \\
\hline CONVErT [10] & & & & & $\checkmark$ & \\
\hline DSL-maps [103] & & & & & $\checkmark$ & \\
\hline Elkamel et al. [37] & & $\checkmark$ & & & & \\
\hline IPSE [40] & & & & & $\checkmark$ & \\
\hline Koschmider et al. $[50,51,71]$ & & & & $\checkmark$ & $\checkmark$ & $\checkmark$ \\
\hline MAGNET [16] & & & & & $\checkmark$ & \\
\hline Paydar et al. $[100,101]$ & & $\checkmark$ & & & & \\
\hline RapMOD $[72,73]$ & & $\checkmark$ & & $\checkmark$ & & $\checkmark$ \\
\hline \multicolumn{7}{|l|}{ No evaluation } \\
\hline \begin{tabular}{|l|}
{$[8,9,17,28,34-36,42,47,60,86,87,91,98,108,110,116,121$,} \\
$126,127,132,137]$
\end{tabular} & & & & & & \\
\hline
\end{tabular}

repeated 5 times with each subset. Similarly, Heinemann [49] used 10-fold cross-validation.

Regardless of the use of datasets, some systems are empirically compared against baselines, which can be naive methods as done by Heinemann [49], who used a RS that suggests the most popular Simulink blocks in libraries. A few cases use existing recommenders built by other researchers, like Li et al. [75] and Deng et al. [31], who compare their approach against two other recommenders for process models. In other cases, the system is evaluated with and without its recommendation component enabled $[99,111]$. Finally, some approaches are evaluated analytically, like Extremo [124,125], whose extensibility is assessed via integration with several thirdparty tools and formats, or PME [99], where the authors built an analytical model to estimate the modelling effort.

Online experiment. Only two of the revised approaches were evaluated using online experiments, both in the context of external projects. ASIMOV [38] was evaluated using a real commercial scenario named Alps Furniture. Two groups of users were asked to co-evolve models either using ASIMOV or manually, and the results were analysed to assess the effort and time reduction achieved when using the tool. The domain modelling 
Table 8 Recommender systems for MDE: evaluation vs metrics

\begin{tabular}{|c|c|c|c|}
\hline Metrics & Offline Experiment & Online Experiment & User Study \\
\hline \multicolumn{4}{|l|}{ Rating Prediction } \\
\hline Ranking Accuracy & $\begin{array}{c}\text { Baya [27] } \\
\text { B-repair [24] } \\
\text { CONVErT [10] } \\
\text { Deng et al. [31] } \\
\text { Heinemann [49] } \\
\text { IntellEdit [93] } \\
\text { Kögel et al. [68, 69] } \\
\text { Li et al. [75] } \\
\text { Refacola [130] } \\
\text { SBPR [64] }\end{array}$ & & $\begin{array}{l}\text { Cerqueira et al. }[26] \\
\text { Elkamel et al. }[37] \\
\text { Paydar et al. }[100,101] \\
\text { RapMOD }[72,73]\end{array}$ \\
\hline \multicolumn{4}{|l|}{ Other Measure } \\
\hline System Performance & \begin{tabular}{|c} 
Baya [27] \\
Deng et al. [31] \\
DiAGEN [82] \\
DIG MDE [92] \\
Extremo [123-125] \\
Li et al. [75] \\
PARMOREL [11,53] \\
PME [99] \\
Refacola [130]
\end{tabular} & ASIMOV [38] & $\begin{array}{c}\text { Baya }[27] \\
\text { Koschmider et al. }[50,51,71] \\
\text { RapMOD [72] }\end{array}$ \\
\hline Usage Satisfaction & & DoMoRe $[5,6]$ & $\begin{array}{c}\text { AXSM [52] } \\
\text { Baya [27] } \\
\text { Cerqueira et al. [26] } \\
\text { CONVErT [10] } \\
\text { DSL-maps [103] } \\
\text { IPSE [40] } \\
\text { Koschmider et al. [50,51,71] } \\
\text { MAGNET [16] }\end{array}$ \\
\hline $\begin{array}{l}\text { Application- } \\
\text { Dependent }\end{array}$ & $\begin{array}{c}\text { AMOR [21] } \\
\text { anATLyzer [119] } \\
\text { Batot et al. [12] } \\
\text { Extremo [123-125] } \\
\text { IntellEdit [93] } \\
\text { Mani et al. [79] } \\
\text { Matikainen et al. [81] } \\
\text { PME [99] } \\
\text { Refacola [130] } \\
\text { Refactory [111] }\end{array}$ & ASIMOV [38] & $\begin{array}{c}\text { anATLyzer }[119] \\
\text { Koschmider et al. }[50,51,71] \\
\text { RapMOD }[72,73]\end{array}$ \\
\hline
\end{tabular}

recommender DoMoRe [5,6] was used in various industrial and research environments, and the user feedback and experience allowed identifying potential aspects for improvement.

User study. There are 12 approaches evaluated with user studies. These typically involve a small group of users that perform some tasks, making it possible to analyse the effectiveness of the users on completing the tasks with and without the recommender, as well as to gather information about user experience via questionnaires [112]. We have identified 3 types of user studies, in which: (i) users perform tasks using the proposed recommender; (ii) users utilise the recommender in an A/B testing setting (i.e. some users perform tasks with the recommender, and some others without it); and (iii) the recommendations are compared to the decisions an expert user would make (i.e. the expert user plays the role of oracle function). The first type of user studies was applied to AXSM [52] to evaluate usage satisfaction; to the RS proposed by Cerqueira et al. [26] to evaluate the usage satisfaction and the accuracy of its sequence diagram recommendations; to CONVErT [10] to get user feedback on the usefulness and usability of the tool to develop transformations aided by interactive recommendations; to DSL-maps [103] to assess the perceived usability and usefulness of its pattern assistant to build meta-models; to IPSE [40] to measure usage satisfaction about its support to help learning UML skills; to MAGNET [16] to get user feedback on the usefulness of the recommendations to learn using AutoFOCUS3; and to RapMOD [72,73] to measure the quality of its graphical model auto-completion recommendations and the reduction of modelling effort. 
Table 9 Public datasets used in the evaluations

\begin{tabular}{|l|l|l|}
\hline Dataset & URL & Paper \\
\hline Alps Furniture meta-model & $\begin{array}{l}\text { https://backus1.uniandes.edu.co/ enar/dokuwiki/doku.php?id= } \\
\text { asimovevaluation }\end{array}$ & {$[38]$} \\
\hline anATLyzer quick fix website (ATL transform.) & http://sanchezcuadrado.es/projects/anatlyzer/quickfixes.html & {$[119]$} \\
\hline ATL transformations Zoo & https://www.eclipse.org/atl/atlTransformations/ & {$[118]$} \\
\hline AtlanMod Ecore Meta-model Zoo & https://web.imt-atlantique.fr/x-info/atlanmod/index.php?title=Ecore & {$[130]$} \\
\hline Extremo website (meta-models) & https://github.com/angel539/extremo/wiki/Performance-Evaluation & {$[123]$} \\
\hline Extremo website (model instances) & https://github.com/angel539/extremo/wiki/Case-Studies & {$[123]$} \\
\hline Google SketchUp (3D models) & https://www.sketchup.com/products/3d-warehouse & {$[81]$} \\
\hline Illinois Semantic Integration Archive & http://pages.cs.wisc.edu/ anhai/wisc-si-archive/ & {$[10]$} \\
\hline Matlab Central File Exchange (Simulink files) & https://www.mathworks.com/matlabcentral/fileexchange/ \\
\hline Model versioning benchmarks & http://www.modelversioning.org/index3899.htm1?option=com_content\& \\
\hline view=article\&id=54\&Itemid=68 & {$[21]$} \\
\hline PARMOREL github (models) & https://github.com/MagMar94/ParmorelRunnable & \\
\hline PARMOREL website (models) & https://ict.hvl.no/project-parmorel/ & {$[53]$} \\
\hline ProB Public Examples Repository & https://www3.hhu.de/stups/downloads/prob/source/ \\
\hline Refactory website (generic model refactorings) & http://www.modelrefactoring.org & {$[11]$} \\
\hline State machine execution contract & http://ecariou.perso.univ-pau.fr/contracts/exec-contract.html & {$[12]$} \\
\hline State machine model and OCL queries & https://github.com/atlanmod/Lazy0cl_StateMachineExample \\
\hline UML-based Web Engineering (UWE) website & https://uwe.pst.ifi.lmu.de/examples.html & {$[12]$} \\
\hline Yahoo! Pipes & http://www.pipes.digital/pipes & {$[100,101]$} \\
\hline
\end{tabular}

The second study type was used by Baya [27] to evaluate (in a crowdsourced user study) whether recommending and weaving mashup model patterns reduces the development time, the number of user interactions and the time between user interactions. In addition, the participants filled-in a questionnaire to evaluate their satisfaction with the tool. Also in this category, Elkamel et al. [37] evaluate the relevance and accuracy of the recommended elements for UML diagrams, and Koschmider et al. [50,51,71] asked two sets of students to create process models with and without recommenders. In the latter case, the authors measured the time spent, the quality of the results and the usage satisfaction.

Finally, two approaches compare their recommendations with the a-priori choices of expert users. The authors of anATLyzer [119] evaluated the usefulness of its quick fixes and the utility of its ranking with respect to the free choices made by two independent developers. Paydar et al. $[100,101]$ used the opinion of experts as the golden standard to evaluate the accuracy of their algorithms to detect behaviour/concepts in use cases, annotate activity diagrams with entities from class diagrams and recommend use cases based on similarity metrics.

Application-independent metrics. The most used ranking accuracy metrics are precision, recall and F-measure [10, $26,27,31,49,64,68,69,72,73,93,100,101]$. Some papers consider additional metrics to evaluate the accuracy of the recommendations, such as mean reciprocal rank (MRR) [100,101]; 11-point interpolated average precision [100, 101]; the average number of recommended alternative solutions per successful recommendation [130]; the hit rate, which is the fraction of correct recommendations in the recommendation list [31]; or relevance and accuracy rates [37].

Several authors measure the performance of their approaches, being time metrics the most common, in particular, the time to compute recommendations $[11,27,53$, $63,73,82,99,123,130]$, and the time spent by the user to perform a task $[27,38,72]$.

Finally, usage satisfaction metrics include mostly feedback from the users after using the system. The feedback is collected informally $[5,6,52]$, by means of questionnaires $[10,16,27,40,103]$ or asking the users to rank the provided recommendations using a Likert scale [26].

Application-dependent metrics. These are metrics specific to MDE activities, such as the number of model editing operations $[38,72,79,99]$, the edit distance between conflict pairs [21], the average number of properties changed per applied quick fix [130], the number of attempts to co-evolve a model [38], the lines of code needed to integrate a meta-modelling tool with the RS $[124,125]$, the number of meta-model constraints fixed in a co-evolution scenario [12], the amount of constraint violations [93], the coverage of a room layout model [81], or the number of valid meta-model/role model matches [111].

Additionally, some metrics are related to the completeness or correctness of the recommendation approach, such as how complete a set of quick fixes is $[79,119]$, the validity of quick fixes or co-evolution actions (they completely remove an error) $[38,79,119,130]$, or the impact of 


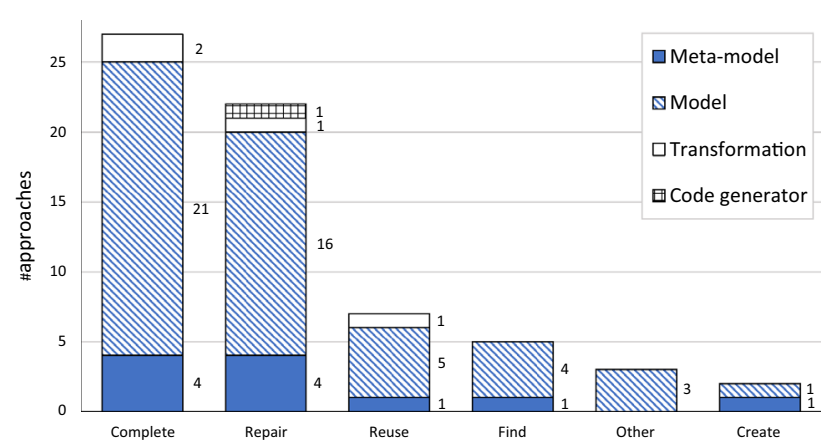

Fig. 9 Distribution of approaches by recommendation purpose

quick fixes (number of problems removed or introduced by their application) $[93,119]$.

\section{Discussion}

This section discusses the results of our systematic mapping in relation to the three RQs posed in the introduction. Section 6.1 answers RQ1 ("In which ways can recommender systems assist in the different tasks within MDE processes?"), Section 6.2 answers RQ2 ("Which recommendation techniques are most commonly used to support MDE tasks, and how are recommenders for MDE evaluated?"), and Section 6.3 answers RQ3 ("What are the main opportunities in recommender systems for MDE solutions?"). Finally, Sect. 6.4 discusses the threats to the validity of our study.

\subsection{RQ1: In which ways can recommender systems assist in the different tasks within MDE processes?}

As discussed in Sect. 5.1, existing RSs for MDE target five main purposes: complete, create, find, repair and reuse. These tasks can be performed over models, meta-models, transformations or code generators.

The graphic in Fig. 9 shows the number of approaches per purpose, stratified by the artefact type. It can be observed that the majority of approaches focus on completion and repair (together, $73.4 \%$ of the approaches), followed by reuse (10.9\%), find (7.8\%), other purposes (4.7\%) and create (3.2\%).

As Fig. 10 shows, most recommenders work over models (76.5\%), followed by meta-models (15.6\%), transformations $(6.2 \%)$ and code generators (1.6\%).

Recommenders with the purpose of completing artefacts typically help in the development of models. For this purpose, some approaches transform partial models into a constraint satisfaction problem or logic programming to obtain a syntactically correct model conformant to its meta-model and

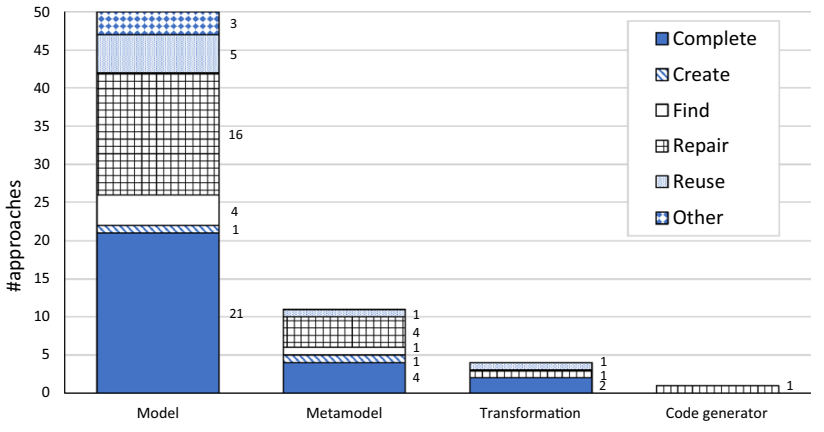

Fig. 10 Distribution of approaches by type of artefact

integrity constraints $[40,82,86,92,126,127,130]$. This may involve adding many elements to the partial model. Instead, other approaches provide finer-grained recommendations for a step-by-step construction of a model. These recommendations are based on similar existing models [34-37,132], model libraries [49], model histories [68,69], knowledge bases [5,6], or a static analysis of the language meta-model [99]. Since meta-models are also models, some approaches can be applied on both of them. Recommenders to complete transformations suggest mappings between source and target elements [10,52].

Recommenders in repair approaches mainly consider models as well. These recommendations assist in repairing inconsistent models using a variety of techniques, such as rules $[38,91,108]$, guidelines [60] or reinforcement learning $[11,53]$. Sometimes, model repair occurs on specific contexts, like meta-model/model co-evolution $[8,38]$ or conflict resolution in model versioning [21]. There is less support to repair meta-models and OCL constraints $[12,28]$, transformations [117-119], and models within code-generation activities [79].

In our study, we have identified numerous languageindependent approaches $[11,34-36,53,68,69,82,86,91,93,98$, 99,108,123-127,130], but most RSs are specific for a modelling language. Figure 11 shows the targeted languages for the language-dependent cases. Most are widely used languages, like UML or process modelling notations, and there are RSs for both structural models (e.g. class diagrams) and behavioural ones (e.g. process models, sequence diagrams and state machines).

It is worth mentioning that there is tool support for $99.2 \%$ of the approaches, though some of them (37.2\%) are prototypes. This demonstrates the feasibility of developing RSs for MDE tasks, but more effort may be needed to increase the number of mature, fully developed tools. As Section 5.2 mentions, most recommenders help in the modelling activity on user demand, but proactive approaches that monitor the user activity to update the recommendations are not uncommon. 


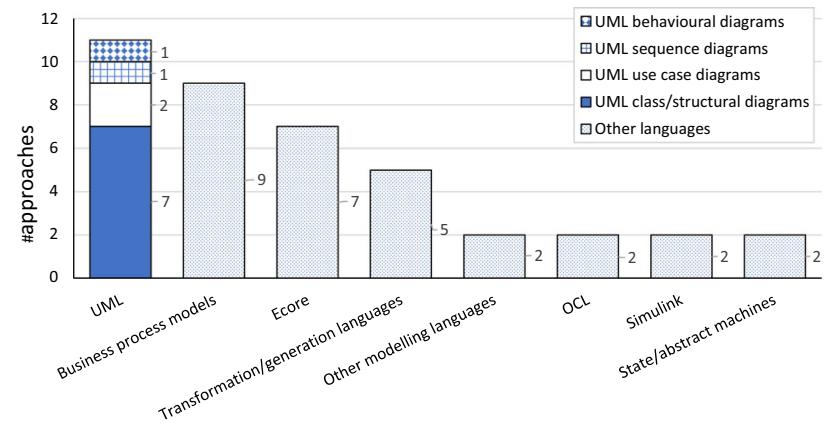

Fig. 11 Distribution of approaches by targeted language

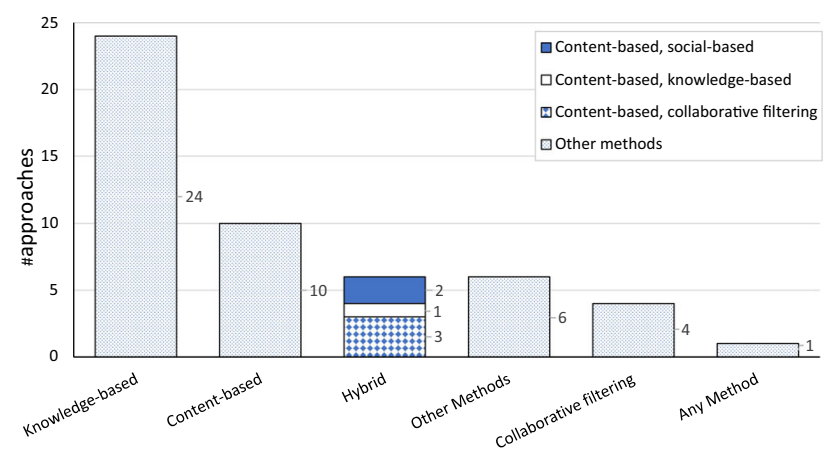

Fig. 12 Distribution of approaches by recommendation method

\subsection{RQ2: Which recommendation techniques are most commonly used to support MDE tasks, and how are recommenders for MDE evaluated?}

Figure 12 shows the recommendation methods used by the studied approaches. Most RSs for MDE are knowledge-based (47\%), followed by content-based (19.6\%), hybrid (11.8\%) and based on collaborative filtering (7.8\%). Among the hybrid approaches, two are content-based and social-based, one is content-based and knowledge-based, and three combine content-based techniques with collaborative filtering. The bar Other Methods refers to ad-hoc methods different from the previous classical recommendation algorithms. Interestingly, there are more RSs applying ad-hoc methods $(11.8 \%)$ than collaborative filtering $(7.8 \%)$. Only one approach is extensible on the recommendation methods and therefore it may potentially support any of them [34-36].

Most of the information to build personalised recommendations is collected implicitly. Only 13 works consider explicit preferences of users, and all but one of those cases use implicit information as well.

An interesting question concerns the relation between recommendation methods and modelling purposes. Table 10 classifies the approaches along these two dimensions (cf. Tables 3 and 6). We can see that content-based methods have been used mostly to complete artefacts, but also to find and reuse them; collaborative filtering has been applied to find,
Table 10 Number of approaches grouped by recommendation purpose and method

\begin{tabular}{lllllll} 
Complete & 6 & 0 & 10 & 4 & 2 & 1 \\
Create & 0 & 0 & 2 & 0 & 0 & 0 \\
Find & 2 & 1 & 0 & 1 & 0 & 0 \\
Repair & 0 & 1 & 12 & 1 & 4 & 0 \\
Reuse & 3 & 0 & 1 & 1 & 0 & 1 \\
Other & 0 & 2 & 1 & 0 & 0 & 0 \\
\hline
\end{tabular}

repair and other purposes; knowledge-based RSs have been extensively used to complete and repair artefacts, as well as for every recommendation purpose in our classification except finding; and other ad-hoc methods have targeted complete and repair.

If we look at the recommendation purpose, we observe that recommenders for completion have used all considered recommendation methods (especially knowledge- and content-based) but collaborative filtering. Creation tasks have only been approached using knowledge-based methods. Recommenders for finding artefacts use collaborative filtering, content-based or hybrid algorithms, but not knowledgebased. Repair has been resolved mostly using knowledgebased methods, but also using collaborative filtering, ad-hoc and hybrid (content-based plus knowledge-based) methods. Finally, reuse has been tackled by content-based, knowledgebased and hybrid (content-based plus collaborative-filtering) methods.

Regarding evaluation, only 32 out of the 51 approaches $(62.7 \%)$ have been evaluated. Offline experiments are the most frequent kind of evaluation [10-12,21,24,27,31,49,53, $64,68,69,75,79,81,82,92,93,99,111,119,123-125,130]$. This may be due to the difficulty to find a relevant number of users with the required level of expertise in modelling and willing to participate in online experiments or user studies. Moreover, some recommenders are implemented for very specific tools developed within research labs, sometimes prototypically, and therefore the tools are neither mainstream nor have a vast number of users. Therefore, while some approaches have been evaluated by means of user studies [10,16,26,27,37,40,50-52,71-73,100,101,103,119], they involve small groups of participants (ranging from 2 to 44$)$, typically students $[10,26,37,40,73,100,101]$, developers/modellers [52,103,119] or more rarely employees $[16,27]$. Online experiments are very scarce $[5,6,38]$.

Offline experiments require data, which sometimes come from public repositories $[10,49,64,81,123,130]$ or companies $[31,75]$. However, in contrast to the programming field, it is difficult to have access to modelling artefacts, especially from industrial projects. For this reason, many authors resort to synthetic datasets created, e.g. via mutation or systematic generation $[24,79,81,93,117-119]$. In other cases, the authors 
evaluate their proposal using artefacts from other papers [24, $68,69]$ or analytically via case studies.

Some of the revised RSs have been evaluated using domain-independent metrics applicable to general RSs, specifically ranking accuracy metrics (mainly precision, recall and F-measure), time metrics and usage satisfaction collected via questionnaires. The advantage is that these metrics are standard and well accepted. As an example, to measure usability (a dimension of usage satisfaction), one could use de-facto standard questionnaires like the System Usability Scale (SUS) [20]. Instead, or in addition, some evaluations have considered metrics specific to MDE tasks-like the number of fixed/violated OCL constraints in a modelor domain-specific notions of completeness or correctness. These metrics are defined ad-hoc for each case.

Finally, we discuss whether some kinds of recommendation tasks are evaluated more than others. We have found that all RSs with the purpose of finding artefacts or fragments have been evaluated (100\%), followed by completion tasks (73.9\% of the RSs helping in completion tasks have been evaluated), repair tasks $(61.1 \%)$, and create $(50 \%)$ and reuse tasks (50\%). RSs targeting repair have been mostly evaluated offline, while recommenders for other purposes have been evaluated using a wider variety of methods.

\subsection{RQ3: What are the main opportunities in recommender systems for MDE solutions?}

This section analyses gaps in the current research, resulting from an analysis of the coverage of the feature model by the proposals. Then, we identify opportunities based on an analysis of the different dimensions of the classification we propose, using both insights from the reviewed papers and our own experience.

Our analysis of the state-of-the-art reveals some gaps in the targeted tasks and artefacts. Most approaches focus on models, a handful on meta-models, very few on transformations, and hardly any on code generators. However, given that MDE fosters the automated processing of models, RSs for transformations and code generators (e.g. recommending completions of the code generation template; suggesting template fragments; or helping to repair faulty generators) would be very useful for the community. Similarly, the purpose of most RSs is completing and repairing models; however, there are few recommendation approaches for finding relevant artefacts, reusing them in a given context and creating artefacts from scratch. For the latter case, we envision RSs proposing initial artefact templates out of higher-level descriptions, maybe defined using natural language. Finally, RSs for structural diagrams are more numerous than those for behavioural diagrams. Developing further RSs for behavioural diagrams would reveal whether behaviour and structure may require different recommenda- tion methods, whether similarity-based recommendation is enough for behavioural diagrams, or whether behavioural diagrams would benefit from semantic comparison (e.g. based on execution) to generate recommendations.

Many of the studied papers present RSs for a specific language or tool (cf. Tables 4 and 5). Such recommenders tackle a single problem and are "hard-wired" into the systems they were designed for. Hence, an open line of research is devising solutions that allow adapting the recommendation algorithms, the users' preferences or the evaluation metrics to the users' needs. In this respect, a reference architecture for intelligent modelling assistance was proposed in [90], and one step in this direction is Hermes [34-36], since this framework permits integrating RSs into tools as well extending the framework with new recommendation methods.

In contrast to the field of programming, one of the main barriers when building RSs for MDE activities is the lack of data that can be used for training the recommenders. There are several initiatives to create repositories of modelling artefacts, both in the MDE [39,76,114] (some listed in Table 9) and BPMN communities [43]. Moreover, dedicated model search engines have been recently proposed [76], which can be used to create datasets of modelling artefacts. However, more efforts to make artefacts public and accessible are required.

Related to the previous point, we are recently witnessing the proposal of low-code development platforms for specific domains, like the creation of data analysis workflows (e.g. RapidMiner, ${ }^{3}$ ) chatbots (e.g. Dialogflow ${ }^{4}$ ) or eventdriven applications (e.g. Node-Red). ${ }^{5}$ These platforms are cloud-based, making it easier for users without a technical background to construct applications by means of graphical languages and forms. Low-code platforms free the user from installing the development tool and deploying the defined applications, since they are used in a web browser. Frequently, low-code platforms form ecosystems where the models created by all users are stored in the platform's repository. This availability of data and users makes low-code platforms the ideal scenario for creating recommender systems, as shown in [55].

As we discussed in Sect. 6.2, a large percentage of the RSs in MDE are knowledge-based or content-based. This differs from the predominance of collaborative filtering and hybrid approaches in the RSs research field [15], where e-commerce (e.g. Amazon, Zalando), leisure (e.g. Netflix, Spotify), tourism (e.g. Booking, Yelp) and social networks (e.g. Facebook, Twitter) are the most widely addressed domains. It is in these domains where large communities of users provide feedback - mainly in the form of numeric

\footnotetext{
3 https://rapidminer.com/.

${ }^{4}$ https://cloud.google.com/dialogflow.

5 https://nodered.org/.
} 
ratings and textual reviews-which is exploited to find user similarities valuable for generating effective personalised recommendations. Following this trend, there are plenty of opportunities to research on how to exploit further collaborative filtering in MDE, for instance, via model and code sharing platforms. Moreover, the few revised works that apply collaborative filtering to MDE neglect long-term users' preferences, hence this stands as a problem worth investigating as well.

Regarding the evaluation of recommendations, Sect. 6.2 shows that only two approaches $[5,6,38]$ report online experiments in real settings, and only one of them [38] uses A/B testing, as commonly done in the online evaluation of information retrieval and filtering approaches. This evaluation methodology not only allows assessing the performance of certain recommendation functionality with users at a large scale, but also its real effectiveness in a non-controlled scenario where contextual conditions arise. In contrast, most approaches were evaluated via offline experiments. In these cases, using public datasets and following standard evaluation methodologies are essential to ensure reproducibility and ease advances in the field. Except for a few cases $[27,119,123]$, we have observed a general lack of reproducibility of the reported experiments. In line with the current open science movement [83], we believe that disclosing replication packages (containing the raw data and all necessary scripts for their analysis) is the way forward in this area. By making datasets available, the creation of new RSs as well as their comparison and improvement is facilitated.

In addition to generic recommendation accuracy and system performance metrics (cf. Table 7), we envision the formal definition and generalisation of metrics oriented to particular MDE tasks (i.e. complete, create, find, repair and reuse) as a relevant research challenge. As our study reveals, the literature already presents ad-hoc metrics, such as the number of model editing operations to evaluate model completion $[72,99]$ or the number of constraint violations to assess the correctness of model repair [93]. However, there is room for designing and reporting more general, well established task-specific measures that would allow comparing distinct recommendation methods.

Related to user experience, an important success factor of RSs is how they integrate within the MDE tool $[1,89,90]$. For notations in the business process modelling domain, some studies investigate how to present recommendations [66], and surveys on the preferred ways to display recommendations in graphical modelling have been conducted as well [36]. However, more usability studies are required to understand the most effective, user-friendly ways to present recommendations for different styles of modelling languages and tasks.

With respect to other research trends on RSs, we highlight recommendation explainability [133] and group-oriented recommendation [80] as two directions of potential interest which, according to our review, have not been addressed yet in the MDE area, but are being extensively investigated by the RS community. On the one hand, explaining to the user the reasons for which recommendations are presented, as well as the potential benefits of the recommendations for the task at hand, can increase the user engagement and trust on the system, among other aspects [133]. On the other hand, there are cooperative tasks and environments that provide recommendations to a group of people, and consequently have to take individual preferences and constraints into account. In this context, the chosen methods for aggregating user models and generating consensus recommendations have to be complemented with an appropriate (collaborative) evaluation [80].

Lastly, although it is out of the scope of this study, we want to mention an open research issue related to the development process of RSs. We have observed that most RSs have been developed by hand from scratch, and very few works have investigated the application of MDE to assist in the design, implementation and evaluation of a RS for a given problem. The development of a RS and its integration into a tool undoubtedly requires a high effort, as noted in [90]. This makes the construction of RSs for DSLs-which typically have a smaller user community than languages like UML-less cost-effective. Therefore, methods for automating the construction of RSs for modelling languages, like those proposed in $[7,129]$, could be very useful for the MDE community.

\subsection{Threats to validity}

Some factors may threat the validity of our study. First, we might have missed some papers due to the query we have used. To mitigate this threat, we tested several versions of the query, confirming that papers we knew were relevant appeared in the query results.

A related threat is that some relevant papers might not be indexed in the databases considered for our query. To mitigate this risk, we performed a final process of snowballing [138] to consider further relevant papers not included in the query results.

In the screening process, we might have erroneously left some relevant papers out. To mitigate this risk, each paper was independently checked by the four authors of the study and was added to the second screening phase if one of them considered it relevant. In this second phase, it was read in full detail.

Finally, there is a thin line dividing the systems that can be considered to provide recommendations, with respect to others that just offer some kind of automated analysis. This situation is exacerbated by the fact that some systems rely on highly specialised algorithms which are non-standard in the RS literature. In our review, we included those systems that provide recommendations and assistance to the user to 
choose a small set of items over a large set of possibilities, or which consider implicit or explicit users' preferences. When in doubt, we included the system in the study.

\section{Summary}

In this paper, we have presented a systematic mapping review of existing research works on RSs for MDE. We have classified those works along four main dimensions (domain, tooling, recommendation and evaluation) characterised by means of feature models.

The review has allowed answering three research questions. First, we have seen that current RSs mainly target model completion and repair. Second, the most used recommendation methods in MDE are knowledge-based and content-based. Finally, we have identified research gaps and opportunities in the area, like implementing RSs to help in developing transformations and code generators, finding and reusing artefacts and creating artefacts from scratch. We encourage the community to pick these challenges to improve the current MDE practice and tooling.

Acknowledgements We thank the reviewers for their useful comments. This work has been funded by the European Union's Horizon 2020 research and innovation programme under the Marie Skłodowska-Curie Grant Agreement No. 813884 (Lowcomote [134]), by the Spanish Ministry of Science (projects MASSIVE, RTI2018-095255-B-I00, and FIT, PID2019-108965GB-I00) and by the R\&D programme of Madrid (Project FORTE, P2018/TCS-4314).

Funding Open Access funding provided thanks to the CRUE-CSIC agreement with Springer Nature.

Open Access This article is licensed under a Creative Commons Attribution 4.0 International License, which permits use, sharing, adaptation, distribution and reproduction in any medium or format, as long as you give appropriate credit to the original author(s) and the source, provide a link to the Creative Commons licence, and indicate if changes were made. The images or other third party material in this article are included in the article's Creative Commons licence, unless indicated otherwise in a credit line to the material. If material is not included in the article's Creative Commons licence and your intended use is not permitted by statutory regulation or exceeds the permitted use, you will need to obtain permission directly from the copyright holder. To view a copy of this licence, visit http://creativecomm ons.org/licenses/by/4.0/.

\section{References}

1. Abrahão, S., Bourdeleau, F., Cheng, B.H.C., Kokaly, S., Paige, R.F., Störrle, H., Whittle, J.: User experience for modeldriven engineering: Challenges and future directions. In: 20th ACM/IEEE International Conference on Model Driven Engineering Languages and Systems, MoDELS, pp. 229-236. IEEE Computer Society (2017)

2. Acceleo. https://www.eclipse.org/acceleo/ (2020)
3. Adomavicius, G., Tuzhilin, A.: Toward the next generation of recommender systems: a survey of the state-of-the-art and possible extensions. IEEE Trans. Knowl. Data Eng. 17(6), 734-749 (2005)

4. Adomavicius, G., Tuzhilin, A.: Context-aware recommender systems. In: Recommender Systems Handbook, pp. 217-253. Springer (2011)

5. Agt-Rickauer, H., Kutsche, R., Sack, H.: Automated recommendation of related model elements for domain models. In: 6th International Conference on Model-Driven Engineering and Software Development (MODELSWARD), Revised Selected Papers, volume 991 of CCIS, pp. 134-158. Springer (2018)

6. Agt-Rickauer, H., Kutsche, R., Sack, H.: DoMoRe-a recommender system for domain modeling. In: 6th International Conference on Model-Driven Engineering and Software Development (MODELSWARD), pp. 71-82. SciTePress (2018)

7. Almonte, L., Cantador, I., Guerra, E., de Lara, J.: Towards automating the construction of recommender systems for lowcode development platforms. In: 1st LowCode Workshop (LowCode@MoDELS),pp. 66:1-66:10. ACM (2020)

8. Anguel, F., Amirat, A., Bounour, N.: Hybrid approach for metamodel and model co-evolution. In: 5th IFIP TC 5 International Conference on Computer Science and its Applications (CIIA), pp. 563-573. Springer (2015)

9. Aquino, E.R., de Saqui-Sannes, P., Vingerhoeds, R.A.: A methodological assistant for use case diagrams. In: 8th International Conference on Model-Driven Engineering and Software Development (MODELSWARD), pp. 227-236. SciTePress (2020)

10. Avazpour, I., Grundy, J., Grunske, L.: Specifying model transformations by direct manipulation using concrete visual notations and interactive recommendations. J. Vis. Lang. Comput. 28, 195211 (2015)

11. Barriga, A., Rutle, A., Heldal, R.: Improving model repair through experience sharing. J. Object Technol. 19(2):13:1-21 (2020)

12. Batot, E., Kessentini, W., Sahraoui, H.A., Famelis, M.: Heuristicbased recommendation for metamodel-OCL coevolution. In: 20th ACM/IEEE International Conference on Model Driven Engineering Languages and Systems (MoDELS), pp. 210-220. IEEE Computer Society (2017)

13. Baudry, B., Ghosh, S., Fleurey, F., France, R.B., Traon, Y.L., Mottu, J.: Barriers to systematic model transformation testing. Commun. ACM 53(6), 139-143 (2010)

14. Bellogín, A., Cantador, I., Castells, P.: A comparative study of heterogeneous item recommendations in social systems. Inf. Sci. 221, 142-169 (2013)

15. Berkovsky, S., Cantador, I., Tikk, D.: Collaborative Recommendations: Algorithms, Practical Challenges and Applications. World Scientific (2018)

16. Bin Abid, S., Mahajan, V., Lucio, L.: Machine learning for learnability of MDD tools. In: 31st International Conference on Software Engineering and Knowledge Engineering (SEKE), pp. 355-468 (2019)

17. Bobek, S., Baran, M., Kluza, K., Nalepa, G.J.: Application of bayesian networks to recommendations in business process modeling. In: Workshop AI Meets Business Processes co-located with AI*IA, volume 1101 of CEUR Workshop Proceedings, pp. 41-50 (2013)

18. Borg, M., Wnuk, K., Regnell, B., Runeson, P.: Supporting change impact analysis using a recommendation system: an industrial case study in a safety-critical context. IEEE Trans. Softw. Eng. 43(7), 675-700 (2017)

19. Brambilla, M., Cabot, J., Wimmer, M.: Model-Driven Software Engineering in Practice, 2nd edn. Synthesis Lectures on Software Engineering. Morgan \& Claypool Publishers (2017)

20. Brooke, J., et al.: SUS-a quick and dirty usability scale. Usab. Eval. Ind. 189(194), 4-7 (1996) 
21. Brosch, P., Seidl, M., Kappel, G.: A recommender for conflict resolution support in optimistic model versioning. In: ACM SIGPLAN Conference on Object-Oriented Programming, Systems, Languages, and Applications, SPLASH/OOPSLA Companion, pp. 43-50. ACM (2010)

22. Burke, R.: Knowledge-based recommender systems. Encycl. Libr. Inf. Syst. 69(Supplement 32), 175-186 (2000)

23. Burke, R.: Hybrid recommender systems: survey and experiments. User Model. User-Adap. Interact. 12(4), 331-370 (2002)

24. Cai, C., Sun, J., Dobbie, G.: Automatic B-model repair using model checking and machine learning. Autom. Softw. Eng. 26(3), 653-704 (2019)

25. Cawley, G.C., Talbot, N.L.C.: On over-fitting in model selection and subsequent selection bias in performance evaluation. J. Mach. Learn. Res. 11, 2079-2107 (2010)

26. Cerqueira, T., Ramalho, F., Marinho, L.B.: A content-based approach for recommending UML sequence diagrams. In: 28th International Conference on Software Engineering and Knowledge Engineering (SEKE), pp. 644-649 (2016)

27. Chowdhury, S.R., Daniel, F., Casati, F.: Recommendation and weaving of reusable mashup model patterns for assisted development. ACM Trans. Internet. Technol. 14(2-3), 21:1-21:23 (2014)

28. Clarisó, R., Cabot, J.: Fixing defects in integrity constraints via constraint mutation. In: 11th International Conference on the Quality of Information and Communications Technology (QUATIC), pp. 74-82. IEEE Computer Society (2018)

29. de Lara, J., Vangheluwe, H.: $\mathrm{AToM}^{3}$ : a tool for multi-formalism and meta-modelling. In: 5th International Conference on Fundamental Approaches to Software Engineering (FASE), volume 2306 of Lecture Notes in Computer Science, pp. 174-188. Springer (2002)

30. de Oliveira, M.C., Freitas, D., Bonifácio, R., Pinto, G., Lo, D.: Finding needles in a haystack: leveraging co-change dependencies to recommend refactorings. J. Syst. Softw. 158, 110420 (2019)

31. Deng, S., Wang, D., Li, Y., Cao, B., Yin, J., Wu, Z., Zhou, M.: A recommendation system to facilitate business process modeling. IEEE Trans. Cybern. 47(6), 1380-1394 (2017)

32. Dey, A.K.: Understanding and using context. Pers. Ubiquit. Comput. 5(1), 4-7 (2001)

33. Dwyer, M. B., Avrunin, G. S., Corbett, J. C.: Patterns in property specifications for finite-state verification. In: 21st International Conference on Software Engineering (ICSE), pp. 411-420. ACM (1999)

34. Dyck, A., Ganser, A., Lichter, H.: Enabling model recommenders for command-enabled editors. In: 1st International Workshop on Model-driven Engineering By Example (MDEBE@MoDELS), volume 1104 of CEUR Workshop Proceedings, pp. 12-21 (2013)

35. Dyck, A., Ganser, A., Lichter, H.: A framework for model recommenders-requirements, architecture and tool support. In: 2nd International Conference on Model-Driven Engineering and Software Development (MODELSWARD), pp. 282-290. SciTePress (2014)

36. Dyck, A., Ganser, A., Lichter, H.: On designing recommenders for graphical domain modeling environments. In: 2nd International Conference on Model-Driven Engineering and Software Development (MODELSWARD), pp. 291-299. SciTePress (2014)

37. Elkamel, A., Gzara, M., Ben-Abdallah, H.: An UML class recommender system for software design. In: 13th IEEE/ACS International Conference of Computer Systems and Applications (AICCSA), pp. 1-8. IEEE Computer Society (2016)

38. Florez, H., Sánchez, M. E., Villalobos, J., Vega, G.: Coevolution assistance for enterprise architecture models. In: 6th International Workshop on Models and Evolution (ME@ MoDELS), pp. 27-32. ACM (2012)

39. France, R.B., Bieman, J.M., Mandalaparty, S.P., Cheng, B.H.C., Jensen, A.C.: Repository for model driven development (remodd).
In: 34th International Conference on Software Engineering (ICSE), pp. 1471-1472. IEEE Computer Society (2012)

40. Garbe, H.: Intelligent assistance in a problem solving environment for UML class diagrams by combining a generative system with constraints. In: eLearning, IADIS (2012)

41. Gasparic, M., Janes, A.: What recommendation systems for software engineering recommend: a systematic literature review. J. Syst. Softw. 113, 101-113 (2016)

42. Gomes, P.: Software design retrieval using bayesian networks and wordnet. In: 7th European Conf. on Advances in Case-Based Reasoning (ECCBR), volume 3155 of Lecture Notes in Computer Science, pp. 184-197. Springer (2004)

43. Großkopf, A., Brunnert, J., Wehrmeyer, S., Weske, M.: Bpmncommunity.org: a forum for process modeling practitioners - A data repository for empirical BPM research. In: Business Process Management Workshops, BPM, volume 43 of Lecture Notes in Business Information Processing, pp. 525-528. Springer (2010)

44. Guerra, E., de Lara, J., Wimmer, M., Kappel, G., Kusel, A., Retschitzegger, W., Schönböck, J., Schwinger, W.: Automated verification of model transformations based on visual contracts. Autom. Softw. Eng. 20(1), 5-46 (2013)

45. Gunawardana, A., Shani, G.: Evaluating recommender systems. In: Recommender Systems Handbook, pp. 265-308. Springer (2015)

46. Guy, I.: Social recommender systems. In: Recommender Systems Handbook, pp. 511-543. Springer (2015)

47. Hayashi, S., YiBing, P., Sato, M., Mori, K., Sejeon, S., Haruna, S.: Test driven development of UML models with SMART modeling system. In: 7th International Conference on The Unified Modelling Language: Modelling Languages and Applications (UML), volume 3273 of Lecture Notes in Computer Science, pp. 395-409. Springer (2004)

48. He, X., Liao, L., Zhang, H., Nie, L., Hu, X., Chua, T.-S.: Neural collaborative filtering. In: 26th International Conference on the World-Wide Web (WWW), pp. 173-182 (2017)

49. Heinemann, L.: Facilitating reuse in model-based development with context-dependent model element recommendations. In: 3rd International Workshop on Recommendation Systems for Software Engineering (RSSE), pp. 16-20. IEEE (2012)

50. Hornung, T., Koschmider, A., Lausen, G.: Recommendation based process modeling support: method and user experience. In: 27th International Conference on Conceptual Modeling (ER), volume 5231 of Lecture Notes in Computer Science, pp. 265-278. Springer (2008)

51. Hornung, T., Koschmider, A., Oberweis, A.: A recommender system for business process models. Inf. Technol., Syst. 47, 13801394 (2009)

52. Huh, J., Grundy, J.C., Hosking, J.G., Li, K.N., Amor, R.: Integrated data mapping for a software meta-tool. In: 20th Australian Software Engineering Conference (ASWEC), pp. 111-120. IEEE Computer Society (2009)

53. Iovino, L., Barriga, A., Rutle, A., Heldal, R.: Model repair with quality-based reinforcement learning. J. Object Technol. 19(2):17:1-21 (2020)

54. Jackson, D.: Software Abstractions-Logic, Language, and Analysis. MIT Press (2006). http://alloytools.org/

55. Jannach, D., Jugovac, M., Lerche, L.: Supporting the design of machine learning workflows with a recommendation system. ACM Trans. Interact. Intell. Syst. 6(1), 8:1-8:35 (2016)

56. Jannach, D., Zanker, M., Felfernig, A., Friedrich, G.: Recommender Systems-An Introduction. Cambridge University Press (2010)

57. Jézéquel, J., Combemale, B., Barais, O., Monperrus, M., Fouquet, F.: Mashup of metalanguages and its implementation in the Kermeta language workbench. Softw. Syst. Model. 14(2), 905-920 (2015) 
58. Jiang, H., Zhang, J., Li, X., Ren, Z., Lo, D., Wu, X., Luo, Z.: Recommending new features from mobile app descriptions. ACM Trans. Softw. Eng. Methodol. 28(4), 22:1-22:29 (2019)

59. Jouault, F., Allilaire, F., Bézivin, J., Kurtev, I.: ATL: a model transformation tool. Sci. Comput. Progr. 72(1-2), 31-39 (2008)

60. Kahloun, F., Ghannouchi, S.A.: Improvement of quality for business process modeling driven by guidelines. In: 22 nd International Conference on Knowledge-Based and Intelligent Information \& Engineering Systems (KES), volume 126 of Procedia Computer Science, pp. 39-48. Elsevier (2018)

61. Kang, K., Cohen, S., Hess, J., Novak, W., Peterson, A.: Featureoriented domain analysis (FODA) feasibility study. Technical Report CMU/SEI-90-TR-021, Software Engineering Institute, Carnegie Mellon University, Pittsburgh, PA (1990)

62. Kelly, S., Tolvanen, J.: Domain-Specific Modeling-Enabling Full Code Generation. Wiley (2008)

63. Khider, H., Hammoudi, S., Benna, A., Meziane, A.: Social business process model recommender: An MDE approach. In: 5th International Conference on Social Networks Analysis, Management and Security (SNAMS), pp. 106-113. IEEE (2018)

64. Khider, H., Hammoudi, S., Meziane, A.: Business process model recommendation as a transformation process in MDE: conceptualization and first experiments. In: 8th International Conference on Model-Driven Engineering and Software Development (MODELSWARD), pp. 65-75. SciTePress (2020)

65. Kim, M.C., Chen, C.: A scientometric review of emerging trends and new developments in recommendation systems. Scientometrics 104(1), 239-263 (2015)

66. Kluza, K., Baran, M., Bobek, S., Nalepa, G.J.: Overview of recommendation techniques in business process modeling. In: Proceedings of 9th Workshop on Knowledge Engineering and Software Engineering (KESE9), volume 1070 of CEUR Workshop Proceedings. CEUR-WS.org (2013)

67. Knijnenburg, B.P., Willemsen, M.C.: Evaluating recommender systems with user experiments. In: Recommender Systems Handbook, pp. 309-352. Springer (2015)

68. Kögel, S.: Recommender system for model driven software development. In: 11th Joint Meeting on Foundations of Software Engineering (ESEC/FSE), pp. 1026-1029. ACM (2017)

69. Kögel, S., Groner, R., Tichy, M.: Automatic change recommendation of models and meta models based on change histories. In: 10th Workshop on Models and Evolution (ME@MoDELS), volume 1706 of CEUR Workshop Proceedings, pp. 14-19 (2016)

70. Koren, Y., Bell, R.: Advances in collaborative filtering. In: Recommender Systems Handbook, pp. 77-118. Springer (2015)

71. Koschmider, A., Hornung, T., Oberweis, A.: Recommendationbased editor for business process modeling. Data Knowl. Eng. 70(6), 483-503 (2011)

72. Kuschke, T., Mäder, P.: RapMOD - in situ auto-completion for graphical models: poster. In: 39th International Conference on Software Engineering (ICSE), Companion Volume, pp. 303-304. IEEE Computer Society (2017)

73. Kuschke, T., Mäder, P., Rempel, P.: Recommending autocompletions for software modeling activities. In: 16th International Conference on Model-Driven Engineering Languages and Systems (MoDELS), volume 8107 of Lecture Notes in Computer Science, pp. 170-186. Springer (2013)

74. Ledeczi, A., Maroti, M., Bakay, A., Karsai, G., Garrett, J., Thomason, C., Nordstrom, G., Sprinkle, J., Volgyesi, P.: The generic modeling environment. In: Workshop on Intelligent Signal Processing, vol. 17, p. 1 (2001)

75. Li, Y., Cao, B., Xu, L., Yin, J., Deng, S., Yin, Y., Wu, Z.: An efficient recommendation method for improving business process modeling. IEEE Trans. Ind. Inf. 10(1), 502-513 (2014)

76. López, J.A.H., Cuadrado, J.S.: MAR: a structure-based search engine for models. In: ACM/IEEE 23rd International Conference on Model Driven Engineering Languages and Systems (MoDELS), pp. 57-67. ACM (2020)

77. Lops, P., De Gemmis, M., Semeraro, G.: Content-based recommender systems: State of the art and trends. In: Recommender Systems Handbook, pp. 73-105. Springer (2011)

78. Maki, S., Kpodjedo, S., Boussaidi, G.E.: Context extraction in recommendation systems in software engineering: a preliminary survey, pp. 151-160. In: IBM Corp (2015)

79. Mani, S., Sinha, V.S., Dhoolia, P., Sinha, S.: Automated support for repairing input-model faults. In: 25th IEEE/ACM International Conference on Automated Software Engineering (ASE), pp. 195204. ACM (2010)

80. Masthoff, J.: Group recommender systems: Combining individual models. In: Recommender Systems Handbook, pp. 677-702. Springer (2011)

81. Matikainen, P., Furlong, P.M., Sukthankar, R., Hebert, M.: Multiarmed recommendation bandits for selecting state machine policies for robotic systems. In: 2013 IEEE International Conference on Robotics and Automation (ICRA), pp. 4545-4551. IEEE (2013)

82. Mazanek, S., Minas., M.: Business process models as a showcase for syntax-based assistance in diagram editors. In: 12th International Conference on Model Driven Engineering Languages and Systems (MoDELS), volume 5795 of Lecture Notes in Computer Science, pp. 322-336. Springer (2009)

83. Méndez, D., Graziotin, D., Wagner, S., Seibold, H.: Open science in software engineering. In: Contemporary Empirical Methods in Software Engineering, pp. 477-501. Springer (2020)

84. Miller, G.A.: WordNet: A lexical database for English. Commun. ACM 38(11), 39-41 (1995)

85. MOF 2.5.1. https://www.omg.org/mof/ (2016)

86. Moha, N., Sen, S., Faucher, C., Barais, O., Jézéquel, J.: Evaluation of Kermeta for solving graph-based problems. Int. J. Softw. Tools Technol. Transfer 12(3-4), 273-285 (2010)

87. Muram, F.U., Gallina, B., Rodriguez, L.G.: Preventing omission of key evidence fallacy in process-based argumentations. In: 11th International Conference on the Quality of Information and Communications Technology (QUATIC), pp. 65-73. IEEE Computer Society (2018)

88. Muslu, K., Brun, Y., Holmes, R., Ernst, M.D., Notkin, D.: Speculative analysis of integrated development environment recommendations. In: 27th Annual ACM SIGPLAN Conf. on Object-Oriented Programming, Systems, Languages, and Applications (OOPSLA), pp. 669-682. ACM (2012)

89. Mussbacher, G., Combemale, B., Abrahão, S., Bencomo, N., Burgueño, L., Engels, G., Kienzle, J., Kühne, T., Mosser, S., Sahraoui, H.A., Weyssow, M.: Towards an assessment grid for intelligent modeling assistance. In: 23rd International Conference on Model Driven Engineering Languages and Systems, Companion Proceedings, pp. 48:1-48:10. ACM (2020)

90. Mussbacher, G., Combemale, B., Kienzle, J., Abrahão, S., Ali, H., Bencomo, N., Búr, M., Burgueño, L., Engels, G., Jeanjean, P., Jézéquel, J., Kühne, T., Mosser, S., Sahraoui, H.A., Syriani, E., Varró, D., Weyssow, M.: Opportunities in intelligent modeling assistance. Softw. Syst. Model. 19(5), 1045-1053 (2020)

91. Nassar, N., Radke, H., Arendt, T.: Rule-based repair of EMF models: an automated interactive approach. In: 10th International Conference on Theory and Practice of Model Transformation (ICMT), volume 10374 of Lecture Notes in Computer Science, pp. 171-181. Springer (2017)

92. Nechypurenko, A., Wuchner, E., White, J., Schmidt, D.C.: Applying model intelligence frameworks for deployment problem in real-time and embedded systems. In: Models in Software Engineering, Workshops and Symposia at MoDELS'06, Reports and Revised Selected Papers, volume 4364 of Lecture Notes in Computer Science, pp. 143-151. Springer (2006) 
93. Neubauer, P., Bill, R., Mayerhofer, T., Wimmer, M.: Automated generation of consistency-achieving model editors. In: IEEE 24th International Conference on Software Analysis, Evolution and Reengineering (SANER), pp. 127-137. IEEE Computer Society (2017)

94. Nguyen, P.T., Rocco, J.D., Ruscio, D.D., Ochoa, L., Degueule, T., Penta., M.D.: FOCUS: a recommender system for mining API function calls and usage patterns. In: 41st International Conference on Software Engineering (ICSE), pp. 1050-1060. IEEE/ACM (2019)

95. Nguyen, P.T., Rocco, J.D., Ruscio, D.D., Penta, M.D.: CrossRec: supporting software developers by recommending third-party libraries. J. Syst. Softw. 161, 110460 (17 pages) (2020)

96. Ning, X., Desrosiers, C., Karypis, G.: A comprehensive survey of neighborhood-based recommendation methods. In: Recommender Systems Handbook, pp. 37-76. Springer (2015)

97. OCL. http://www.omg.org/spec/OCL/ (2014)

98. Ohrndorf, M., Pietsch, C., Kelter, U., Kehrer, T.: ReVision: a tool for history-based model repair recommendations. In: 40th International Conference on Software Engineering (ICSE), Companion Proceeedings, pp. 105-108. ACM (2018)

99. Pati, T., Kolli, S., Hill, J.H.: Proactive modeling: a new model intelligence technique. Softw. Syst. Model. 16(2), 499-521 (2017)

100. Paydar, S., Kahani, M.: A semantic web enabled approach to reuse functional requirements models in web engineering. Autom. Softw. Eng. 22(2), 241-288 (2015)

101. Paydar, S., Kahani, M.: A semi-automated approach to adapt activity diagrams for new use cases. Inf. Softw. Technol. 57, 543-570 (2015)

102. Pazzani, M.J.: A framework for collaborative, content-based and demographic filtering. Artif. Intell. Rev. 13(5-6), 393-408 (1999)

103. Pescador, A., de Lara, J.: DSL-maps: from requirements to design of domain-specific languages. In: 31st IEEE/ACM International Conference on Automated Software Engineering (ASE), pp. 438443. ACM (2016)

104. Petersen, K., Feldt, R., Mujtaba, S., Mattsson, M.: Systematic mapping studies in software engineering. In: 12th International Conference on Evaluation and Assessment in Software Engineering, EASE, Workshops in Computing. BCS (2008)

105. Petersen, K., Vakkalanka, S., Kuzniarz, L.: Guidelines for conducting systematic mapping studies in software engineering: an update. Inf. Softw. Technol. 64, 1-18 (2015)

106. Quijano-Sánchez, L., Cantador, I., Cortés-Cediel, M.E., Gil, O.: Recommender systems for smart cities. Inf. Syst. 92, 101545 (2020)

107. QVT 1.3. http://www.omg.org/spec/QVT/ (2016)

108. Rabbi, F., Lamo, Y., Yu, I.C., Kristensen, L.M.: A diagrammatic approach to model completion. In: 4th Workshop on the Analysis of Model Transformations (AMT@MoDELS), volume 1500 of CEUR Workshop Proceedings, pp. 56-65 (2015)

109. Rabbi, F., Lamo, Y., Yu, I.C., Kristensen, L.M.: Diagrammatic development of domain specific modelling languages with webdpf. Int. J. Inf. Syst. Model. Des. 7(3), 93-114 (2016)

110. Rangiha, M.E., Comuzzi, M., Karakostas, B.: Role and task recommendation and social tagging to enable social business process management. In: BPMDS/EMMSAD@CAiSE, volume 214 of Lecture Notes in Business Information Processing, pp. 68-82. Springer (2015)

111. Reimann, J., Seifert, M., Aßmann, U.: On the reuse and recommendation of model refactoring specifications. Softw. Syst. Model. 12(3), 579-596 (2013)

112. Ricci, F., Rokach, L., Shapira, B. (eds.): Recommender Systems Handbook. Springer (2015)

113. Robillard, M.P., Walker, R.J., Zimmermann, T.: Recommendation systems for software engineering. IEEE Softw. 27(4), 80-86 (2010)
114. Rocco, J.D., Ruscio, D.D., Iovino, L., Pierantonio, A.: Collaborative repositories in model-driven engineering. IEEE Softw. 32(3), 28-34 (2015)

115. Rose, L.M., Paige, R.F., Kolovos, D.S., Polack, F.: The Epsilon generation language. In: 4th European Conf. on Model Driven Architecture-Foundations and Applications (ECMDA-FA), volume 5095 of Lecture Notes in Computer Science, pp. 1-16. Springer (2008)

116. Saini, R., Mussbacher, G., Guo, J.L.C., Kienzle, J.: Teaching modelling literacy: An artificial intelligence approach. In: 22nd ACM/IEEE International Conference on Model Driven Engineering Languages and Systems (MoDELS), Companion Proceedings, pp. 714-719. IEEE (2019)

117. Sánchez Cuadrado, J., Guerra, E., de Lara, J.: Quick fixing ATL model transformations. In: 18th ACM/IEEE International Conference on Model Driven Engineering Languages and Systems (MoDELS), pp. 146-155. IEEE Computer Society (2015)

118. Sánchez Cuadrado, J., Guerra, E., de Lara, J.: AnATLyzer: an advanced IDE for ATL model transformations. In: 40th International Conference on Software Engineering (ICSE), Companion Proceedings, pp. 85-88. ACM (2018)

119. Sánchez Cuadrado, J., Guerra, E., de Lara, J.: Quick fixing ATL transformations with speculative analysis. Softw. Syst. Model. 17(3), 779-813 (2018)

120. Sarwar, B., Karypis, G., Konstan, J., Riedl, J.: Item-based collaborative filtering recommendation algorithms. In: 10th International Conference on the World-Wide Web (WWW), pp. 285-295 (2001)

121. Savary-Leblanc, M.: Improving MBSE tools UX with aiempowered software assistants. In: 22nd ACM/IEEE International Conference on Model Driven Engineering Languages and Systems (MoDELS), Companion Volume, pp. 648-652. IEEE (2019)

122. Schmidt, D.C.: Guest editor's introduction: model-driven engineering. Computer 39(2), 25-31 (2006)

123. Segura, Á.M., de Lara, J.: Extremo: an eclipse plugin for modelling and meta-modelling assistance. Sci. Comput. Program. 180, 71-80 (2019)

124. Segura, Á.M., de Lara, J., Neubauer, P., Wimmer, M.: Automated modelling assistance by integrating heterogeneous information sources. Comput. Lang. Syst. Struct. 53, 90-120 (2018)

125. Segura, Á.M., Pescador, A., de Lara, J., Wimmer, M.: An extensible meta-modelling assistant. In: 20th IEEE International Enterprise Distributed Object Computing Conference (EDOC), pp. 1-10. IEEE Computer Society (2016)

126. Sen, S., Baudry, B., Vangheluwe, H.: Domain-specific model editors with model completion. In: Models in Software Engineering, Workshops and Symposia at MoDELS'07, Reports and Revised Selected Papers, volume 5002 of Lecture Notes in Computer Science, pp. 259-270. Springer (2007)

127. Sen, S., Baudry, B., Vangheluwe, H.: Towards domain-specific model editors with automatic model completion. Simulation 86(2), 109-126 (2010)

128. Simulink. https://www.mathworks.com/products/simulink.html (2020)

129. Sipio, C.D., Ruscio, D.D., Nguyen, P.T.: Democratizing the development of recommender systems by means of low-code platforms. In: 1st LowCode Workshop (LowCode@MoDELS), pp. 68:168:9. ACM (2020)

130. Steimann, F., Ulke, B.: Generic model assist. In: 16th International Conference on Model-Driven Engineering Languages and Systems (MoDELS), volume 8107 of Lecture Notes in Computer Science, pp. 18-34. Springer (2013)

131. Steinberg, D., Budinsky, F., Paternostro, M., Merks, E.: EMF: Eclipse Modeling Framework, 2nd edn. Addison-Wesley Professional (2008) 
132. Stephan, M.: Towards a cognizant virtual software modeling assistant using model clones. In: 41st International Conference on Software Engineering: New Ideas and Emerging Results (NIER@ICSE), pp. 21-24. IEEE/ACM (2019)

133. Tintarev, N., Masthoff, J.: Evaluating the effectiveness of explanations for recommender systems. User Model. User-Adap. Int. 22(4-5), 399-439 (2012)

134. Tisi, M., Mottu, J., Kolovos, D.S., de Lara, J., Guerra, E., Ruscio, D.D., Pierantonio, A., Wimmer, M.: Lowcomote: training the next generation of experts in scalable low-code engineering platforms. In: STAF (Co-Located Events), volume 2405 of CEUR Workshop Proceedings, pp. 73-78. CEUR-WS.org (2019)

135. Tsunoda, M., Kakimoto, T., Ohsugi, N., Monden, A., Matsumoto, K.: Javawock: A Java class recommender system based on collaborative filtering. In: 17th International Conference on Software Engineering and Knowledge Engineering (SEKE), pp. 491-497 (2005)

136. UML 2.5.1. https://www.uml.org/ (2017)

137. Witt, S., Feja, S., Speck, A., Hadler, C.: Business application modeler: A process model validation and verification tool. In: IEEE 22nd International Requirements Engineering Conference (RE), pp. 333-334. IEEE Computer Society (2014)

138. Wohlin, C.: Guidelines for snowballing in systematic literature studies and a replication in software engineering. In: 18th International Conference on Evaluation and Assessment in Software Engineering, EASE, pp. 38:1-38:10. ACM (2014)

139. Wohlin, C., Runeson, P., da Mota Silveira Neto, P.A., Engström, E., do Carmo Machado, I., de Almeida, E.S.: On the reliability of mapping studies in software engineering. J. Syst. Softw. 86(10):2594-2610 (2013)

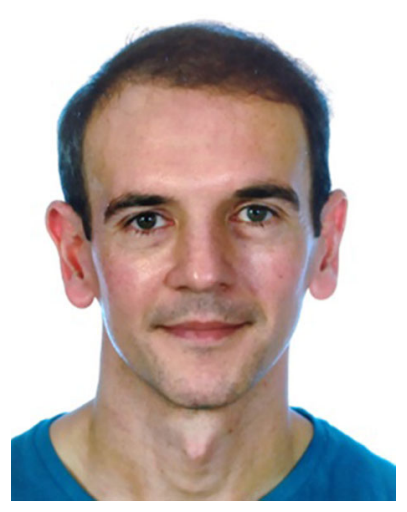

Iván Cantador is Senior Lecturer in Computer Science at Universidad Autónoma de Madrid. His main research lines are in the Recommender Systems field, where he has investigated a wide range of issues related to user modelling, knowledge representation, and processing and mining of usergenerated content. Contact him at ivan.cantador@uam.es, or visit http:// www.eps.uam.es/ cantador

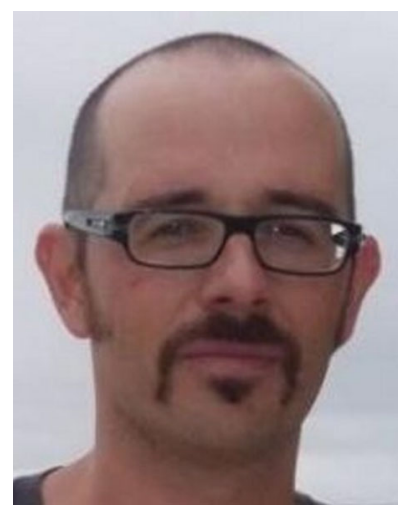

Juan de Lara is Full professor at the computer science department of the Universidad Autónoma de Madrid. Together with E. Guerra, he leads the modelling and software engineering research group. His research interests are in modeldriven engineering and automated software development. Contact him at juan.delara@uam.es, or visit http:// arantxa.ii.uam.es/ jlara/.
Publisher's Note Springer Nature remains neutral with regard to jurisdictional claims in published maps and institutional affiliations.
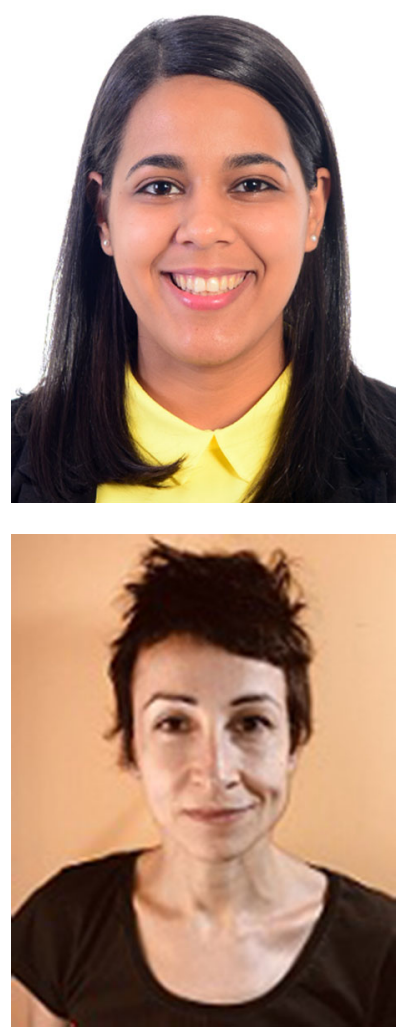

Esther Guerra is Associate Professor at the Computer Science department of the Universidad Autónoma de Madrid. Together with J. de Lara, she leads the modelling and software engineering research group (http://miso. es). She is interested in flexible modelling, meta-modelling, domainspecific languages and model transformation. Contact her at esther.guerra@uam.es, or visit http:// www.eps.uam.es/ eguerra.
Lissette Almonte is a Ph.D's student in Computer Science at the Universidad Autónoma de Madrid and the main developer of DROID. Recommender Systems for Model driven Software Development. Contact her at lissette.almonte@uam.es. 